\title{
PARAMETERIZED UNCERTAINTY MODEL USING A GENETIC ALGORITHM WITH APPLICATION TO AN ELECTRO-HYDRAULIC VALVE CONTROL SYSTEM
}

\author{
A Thesis presented to the Faculty of the Mechanical \\ and Aerospace Engineering Department \\ at the University of Missouri
}

In Partial Fulfillment

of the Requirements for the Degree

Master of Science

\author{
by \\ ZUHENG KANG
}

Dr. Roger Fales, Thesis Supervisor

MAY 2016 
The undersigned have examined the thesis entitled

\section{PARAMETERIZED UNCERTAINTY MODEL USING A GENETIC ALGORITHM WITH APPLICATION TO AN ELECTRO- HYDRAULIC VALVE CONTROL SYSTEM}

presented by Zuheng Kang, a candidate for the degree of Master of Science, and hereby certify that, in their opinion, it is worthy of acceptance.

Professor Roger Fales

Professor Bahaa Kazem

Professor Carmen Chicone 


\section{ACKNOWLEDGEMENTS}

I would like to thank Dr. Roger Fales and Dr. Bahaa Kazem for his advisement and guidance, and for being so supportive throughout this research. It has been a privilege studying and researching under them.

I would like to thank my Father Daniel Kang, and mother Susan King, their continuous supportive to help me finish my academic study in University of Missouri Columbia. 


\section{TABLE OF CONTENTS}

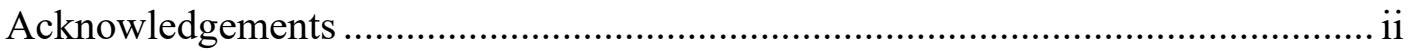

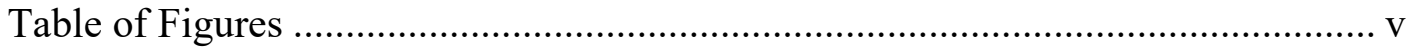

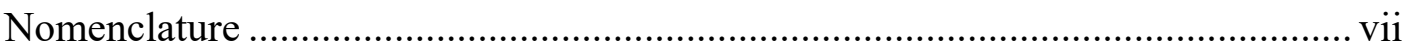

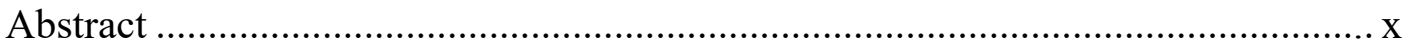

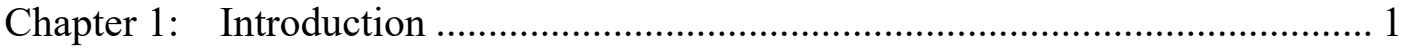

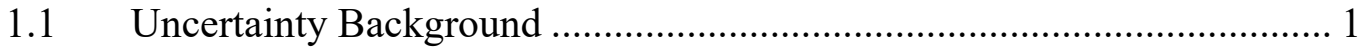

1.2 Previous Works ....................................................................................... 1

1.3 Approach to Adopt GA in Optimization ............................................... 4

$1.4 \quad$ List of Goals and Contributions …...................................................... 4

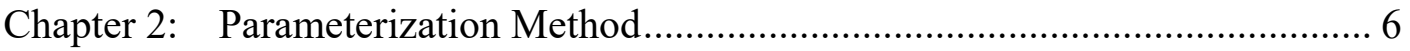

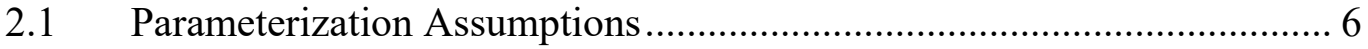

2.2 Parameterizing a Transfer Function ................................................... 6

Chapter 3: Genetic Algorithm Optimization of Uncertainty Model Coefficients 14

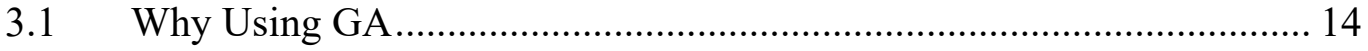

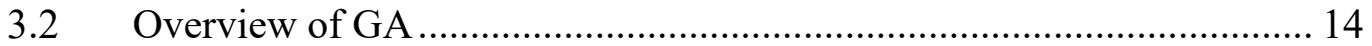

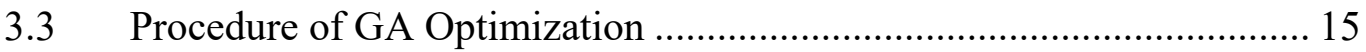

STEP 1. Initialize Genes and Population............................................... 15

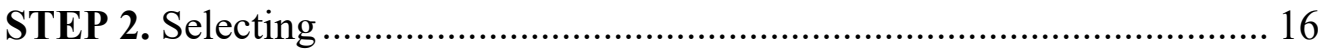

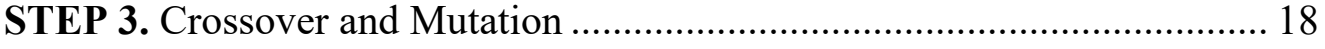


STEP 4. Iteration

STEP 5. Getting the Result

3.4 Test Uncertainty Model............................................................... 19

Chapter 4: Practical Example - Electro-Hydraulic Valve.................................. 22

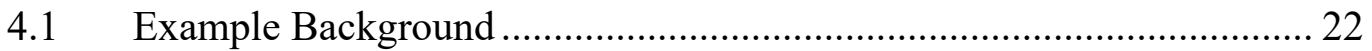

4.2 Close Loop System Analysis....................................................... 22

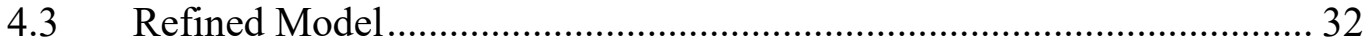

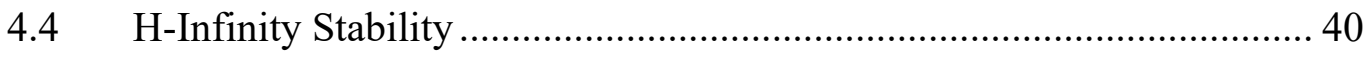

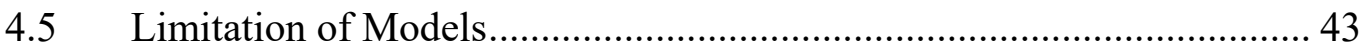

Chapter 5: Dimension Search Conceptual Design ....................................... 45

5.1 Overview of Dimension Search Method ............................................. 45

5.2 Conceptual Design of Dimension Search Algorithm ........................... 46

5.3 Examples and Results of Dimension Search Algorithm ...................... 48

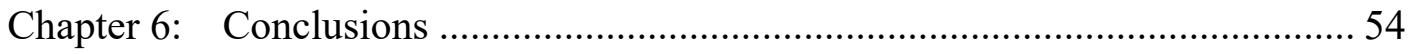

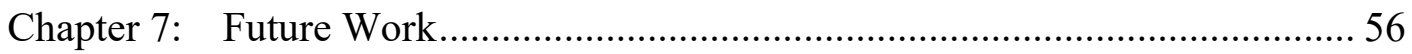

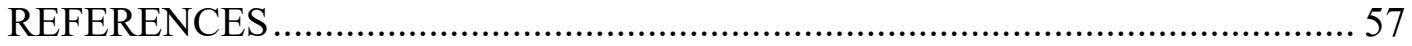

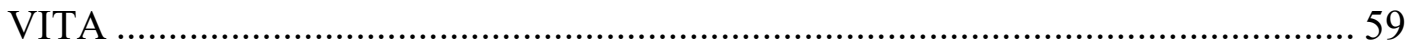




\section{TABLE OF FIGURES}

Figure 2.1 Distance $d_{k}$ for a set of samples..................................................... 12

Figure 3.1 Crossover and Mutation...................................................................... 18

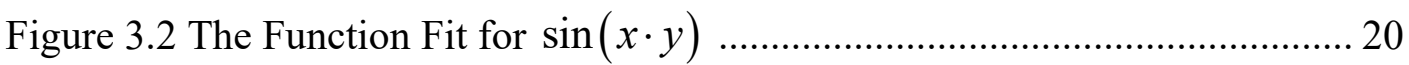

Figure 3.3 Value of Uncertainty Variables ....................................................... 21

Figure 4.1 The Input Chirp Signal and Corresponding Response in Time Domain 23

Figure 4.2 List of Frequency Response of 30 Replicative Valves ......................... 28

Figure 4.3 Frequency response fit (solid line) for transfer function for example data

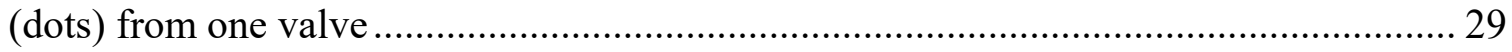

Figure 4.4 GA fit for point cloud in 3-Dimensional View..................................... 30

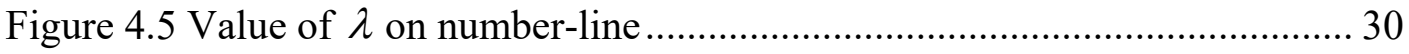

Figure 4.6 Analysis of Feedback Control for Uncertainty Model........................... 31

Figure 4.7 The Closed-loop Poles for uncertainty model ....................................... 31

Figure 4.8 Feasible region for $K$ in a stable close-loop controller ........................ 32

Figure 4.9 The Fitting Transfer Function by Nonlinear Fitting Method.................. 33

Figure 4.10 The Plot of Point Cloud for the Data Set ............................................. 34

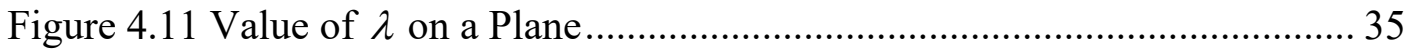

Figure 4.12 The distribution function of the uncertainty variables .......................... 36

Figure 4.13 The Re-Sampled Data Set............................................................. 37

Figure 4.14 The Frequency Response of All the Fitting Data Set ........................... 38

Figure 4.15 The Bode Plot of All the Re-Sampled Data Set.................................. 38 
Figure 4.16 The Closed-loop Poles of the Resampled Transfer Functions at

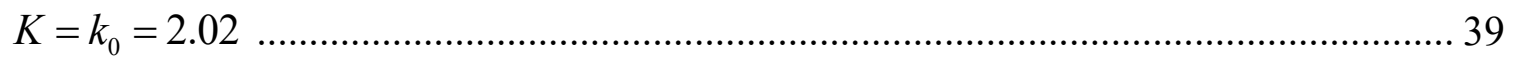

Figure 4.17 The Family of Transfer Functions for Multiplicative Error ................ 41

Figure 4.18 The Transfer Function Fit for Multiplicative Error $W(s)$................ 41

Figure 4.19 Feedback Control with Multiplicative Error Form .......................... 42

Figure 4.20 The Norm Function of the Transfer Function at $K=2.02$................. 42

Figure 4.21 The Norm Function of the Transfer Function at $K=0.53$............... 43

Figure 4.22 The Closed-loop Poles of the Resampled Transfer Functions at

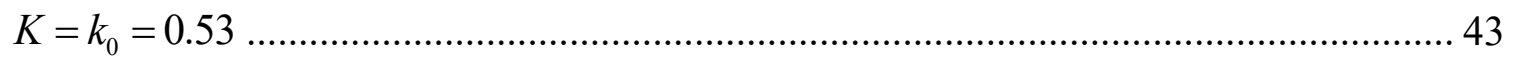

Figure 5.1 Example of 1-D Properties of Data Set ......................................... 46

Figure 5.2 The Dimension Test Geometry ......................................................... 47

Figure 5.3 The Example of 3-D Helix Curve ................................................. 49

Figure 5.4 1-D Property of 3-D Helix Curve .................................................. 49

Figure 5.5 2-D Property of 3-D Helix Curve .................................................... 50

Figure 5.6 3-D Property of 3-D Helix Curve ................................................. 50

Figure 5.7 The Example of 3-D Helix Curve with Less Sample .......................... 51

Figure 5.82 1-D Property of Less Sampled 3-D Helix Curve .............................. 52

Figure 5.9 2-D Property of 3-D Helix Curve .................................................... 52

Figure 5.10 3-D Property of 3-D Helix Curve ............................................... 53 


\section{NOMENCLATURE}

\begin{tabular}{|c|c|c|}
\hline$c$ & $=$ & Each values of constant matrix $\left[\bar{C}^{\prime}\right]$ \\
\hline$R$ & $=$ & $\begin{array}{l}\text { Frequency response } \\
\text { functions for samples }\end{array}$ \\
\hline$\mu$ & $=$ & Mutation coefficient \\
\hline$N(\square)$ & $=$ & Normalization function \\
\hline$\hat{\theta}$ & $=$ & System parameter \\
\hline$\hat{\Theta}$ & $=$ & System parameter set \\
\hline$\|\square\|_{p}$ & $=$ & The $p$ norm function \\
\hline$\delta_{i j}$ & $=$ & $\begin{array}{l}\text { The Cartesian distance of } i^{\text {th }}, j^{\text {th }} \\
\text { nodes }\end{array}$ \\
\hline$\gamma$ & $=$ & $\begin{array}{l}\text { The constant affect the ripple } \\
\text { coefficient }\end{array}$ \\
\hline$\left[\bar{C}^{\prime}\right]$ & $=$ & $\begin{array}{l}\text { The constant matrix after } \hat{\theta} \text { and } \lambda \\
\text { normalization transformation }\end{array}$ \\
\hline$\left[C^{\prime}\right]$ & $=$ & $\begin{array}{l}\text { The constant matrix after } \hat{\theta} \\
\text { normalization transformation }\end{array}$ \\
\hline$[C]$ & $=$ & $\begin{array}{l}\text { The constant matrix for uncertainty } \\
\text { model }\end{array}$ \\
\hline$d$ & $=$ & The distance error - shortest distance \\
\hline
\end{tabular}


and the perturbed system parameters

$\theta$

$t o_{10}(\square)=\quad$ The function convert binary number to decimal number

$v_{i}(\lambda)^{T}=\quad$ The group of uncertainty variables with $i^{\text {th }}$ highest order

$k=\quad$ The index of sample sets from $\hat{\Theta}$

$M_{k}=$ The measurement for $k$ dimension property of point cloud

$\Delta=$ The measurement of small test geometry element

$\hat{\theta}^{\prime} \quad=\quad$ The normalized system parameters

$f^{\prime}=\quad$ The normalized uncertainty model function

$\kappa=\quad$ The number of $\hat{\theta}$ in $\hat{\Theta}$

$O \quad=\quad$ The offspring

$\theta \quad=\quad$ The perturbed system parameters on uncertainty model function $f$

$G \quad=\quad$ The perturbed transfer function with system parameters

$\Pi \quad=\quad$ The probability function

$K=$ The proportional gain of the 
controller

\begin{tabular}{|c|c|c|}
\hline$\Lambda$ & $=$ & The ripple coefficient \\
\hline$P$ & $=$ & The selected parents \\
\hline$D$ & $=$ & The summation of all distance error \\
\hline$W$ & $=$ & $\begin{array}{l}\text { The transfer function fit for } \\
\text { maximum multiplicative error }\end{array}$ \\
\hline$E_{i}$ & $=$ & $\begin{array}{l}\text { The transfer function of } \\
\text { multiplicative Error for all resampled } \\
\text { transfer functions }\end{array}$ \\
\hline$G_{0}$ & $=$ & $\begin{array}{l}\text { The transfer function of nominal } \\
\text { plant }\end{array}$ \\
\hline$\left[T_{\hat{\theta}}\right]$ & $=$ & $\begin{array}{l}\text { The transformation matrix to } \\
\text { normalize system parameters } \hat{\theta}\end{array}$ \\
\hline$\left[T_{\lambda}\right]$ & $=$ & $\begin{array}{l}\text { The transformation matrix to } \\
\text { normalize uncertain variable } \lambda\end{array}$ \\
\hline$f$ & $=$ & The uncertainty model function \\
\hline $\bar{\lambda}$ & $=$ & $\begin{array}{l}\text { The value of uncertainty variables } \\
\text { before normalization transform }\end{array}$ \\
\hline$\lambda$ & $=$ & Uncertainty variables \\
\hline$t_{p}$ & $=$ & Untwisted coefficient \\
\hline FFT & $=$ & Fast Fourier Transformation \\
\hline GA & $=$ & Genetic Algorithm \\
\hline SISO & $=$ & Single Input Single Output \\
\hline
\end{tabular}




\title{
PARAMETERIZED UNCERTAINTY MODEL USING A GENETIC ALGORITHM WITH APPLICATION TO AN ELECTRO-HYDRAULIC VALVE CONTROL SYSTEM
}

\author{
Zuheng Kang
}

Dr. Roger Fales, Thesis Supervisor

\begin{abstract}
Uncertainties in the mechanical system severely increase the complexity of the system. Present the uncertainties can be parameterized by listing the coefficient from the transfer function. The attempt to reduce the uncertainties by capturing the type of data, then reproducing the data with a simple form easy for doing further analysis is the kernel technique in building uncertainty model to transform uncertainties into an easier form. However, since the traditional optimization algorithms proposed by previous researchers may trapped in solving non-convex polynomial function optimization problem, yet genetic algorithm is better to avoid non-convex problem, any discoveries on developing the effective GA (genetic algorithm) with better convergence haves been presented. Because GA contains unpredictable variations, a fine tuning on the algorithm specifically for one type of problem is needed. Focusing on increasing the speed and performance of the algorithm, a series of normalization method is adopted based on the assumption of linear transformation; as well as preventing converging to a false solution (such as the twisted result), series of coefficients are defined. The
\end{abstract}


GA optimization is working by selecting by roulette wheel method with probability weights for the population.

The parameterized uncertainty model presented here are demonstrated from test data for an electro-hydraulic pilot valve control system problem, including the parameterizing an uncertainty class determined from test data for 30 replications of an electro-hydraulic flow control valve. The process of reproducing the data set is then achieved by re-sampling technique with a manipulated probability distribution function. Thereafter this parameterization of the uncertainty used for analyzing the robust stability of a control system for a class of valves is possible.

Keywords: parameterized uncertainty model, genetic algorithm, resampling, dimension search 


\section{CHAPTER 1: INTRODUCTION}

\subsection{Uncertainty Background}

In control systems, variations in the dynamics of plants can create the uncertainties, which make it difficult for the designers to design controllers to control the system. The deviations, which lead to uncertainty, occur due to various conditions, such as manufacturing tolerance in batch fabrication and the different working conditions of the plant. Uncertainty models can be used in analysis of robust stability and uncertainty as well as robust controller synthesis [1]. Often, inefficient and complicated models are used to cover all uncertainties, thus a simpler model of uncertainty is needed.

In order to design robustly stable controllers with better system performance, less conservative approaches to modeling uncertainty would always be preferable. Often uncertainty models are created with the assumption that parameters can vary independently without constraint. In some cases, the relationships between the system parameters are known or can be found, which suggests that models can be formed that do not exaggerate the uncertainties by assuming that parameters can vary independently. However, the significantly challenging part of this problem is to discover a method capturing these relationships between parameters.

\subsection{Previous Works}

Several related approaches have been proposed by different authors. Uncertainty models based on frequency responses have been created for 
applications such as electrohydraulic valves [2]. In the cited work, an uncertainty model was created for a series of 30 valves and differs from the work presented here in that the model of the valve was not parameterized though parametric uncertainty was present. The methods in a work by Sepasi, et. al. is similar to the work presented here in that a parameterized model of uncertainty is found [3]. However, a different method of optimization of the parameterization of the model (the relationship between parameters as well as the number of parameters required) is used in the work presented here.

A method for identifying model parameters and parametric uncertainty for hard disk drives (HDDs) has been presented by Conway et. al.[4]. The authors use modal analysis to find parameters of a set of HDDs. They use avoid redundant parameters by two methods: 1 , combining experimental data directly to extract a low number of parameters, 2 , using a method that optimizes model truncation. A unique optimization process is used to find an approximation of the uncertainty parameters in method 2. The result is a reduced order model with parametric uncertainty with parameters described by a low order approximation that avoids being too conservative. The model and uncertainty are then used in a control design.

Sepasi et. al. presents a method to find an uncertainty model for linear time invariant (LTI) systems[3]. In their work a finite number of rational transfer functions are considered to represent a class of uncertain plants. The transfer functions all have the same order (same number of poles and zeros). The authors present a method to describe the set of transfer functions by a reduced set of 
parameters. In an illustrative example, the number of required parameters is found to be less than the sum of the order of the numerator and denominator. Finding an optimal parametric model was formulated as a non-convex problem. The authors used linear matrix inequalities and nonlinear optimization techniques in their approach to finding the low order parametric model.

Yazdi et. al. [5] presented a controller design procedure, by combining previously developed techniques in parameter uncertainty modeling and multiple robust controller design. The parameter uncertainty modeling technique yields a tight parametric uncertainty set, thus reducing unnecessary conservatism in robust control design method. Yazdi et. al. sampled a number of (Hard Disk Drive) HDDs from the production line. They found a frequency response function for each sampled HDD. Therefore, a set of transfer functions with parametric uncertainties represent the sampled HDDs. Also "intermediate" HDDs would be represented by the set. Correlations found, if any, among coefficient parameter variations would allow the parameterization of the set with a small (reduced) number of uncorrelated uncertain parameters. In their work, the model structure was not automatically determined, but was manually selected.

The connected set was required to represent the intermediate HDDs between the sampled ones in the data set. The reduced number of uncorrelated parameters in the model simplifies the parameterization of the model, leading to both reduced conservatism and reduced computational complexity in subsequent controller design or analysis. 


\subsection{Approach to Adopt GA in Optimization}

In this article, we proposed Genetic Algorithm (GA) to search for a parameterization of the uncertainty in a model. Then, several methods of improving the accuracy and the performance of GA has been suggested. Firstly, the normalization method of fixed order transformation approach has been used. Furthermore, the ripple coefficient, untwisted coefficient, and obvious coefficient were raised to establish the probability coefficient acting as a criterion for the effective selection of the population. Finally, we provide three parameters to describe the convergence of the algorithm. Then, a practical example of the analysis of the stability and robustness of an electrohydraulic valve system is given by applying this uncertainty model. The next chapter followed by a dimension search conceptual design by introducing Cayley-Menger Determinant [6] in order to test the reduced dimension properties within higher dimension space.

\subsection{List of Goals and Contributions}

This thesis is focusing on four goals:

1. To build uncertainty model dealing with reduced dimension point cloud search;

2. Fine tuning of Genetic Algorithm to achieve better performance of searching dimensions;

3. Finding an effective way to parameterize the transfer functions;

4. Finding an effective dimension search algorithm to capture the dimension properties of reduced geometries. 
This thesis has four contributions:

1. Created normalization method of linear transformation to achieve better performance of GA;

2. Established a complex nonlinear fitting optimization algorithm to fit the transfer function model;

3. Resampling process with kernel-mix distribution method has been created;

4. Built a dimension search concept design method to show reduced dimension properties of the point cloud using Cayley-Menger Determinant. 


\section{CHAPTER 2: PARAMETERIZATION METHOD}

\subsection{Parameterization Assumptions}

The system parameters come from the equivalent model of mechanical system. We will assume that, all the system parameters will change continuously as the physical deviation of the system varies, ensuring that each step of a function transformation would be continuous. Although the system parameters can accurately include all uncertainties, the relationships between parameters may contain some rules (or constraint relationships) and therefore reducing the number of parameters can further reduce the uncertainty and complexity. By reducing complexity, the behavior of the system will more clear, simplifying the process of analyzing the closed loop system and designing a controller to control the system.

\subsection{Parameterizing a Transfer Function}

The frequency response for the Single-Input-Single-Output (SISO) model can be achieved by applying Fast Fourier Transform method (FFT) on both the input and output signal, then taking the ratio to manipulate the transfer function form. This can be done for a set of frequency responses creating a limited data set of a finite number of frequency responses. Then, fix a structure for the transfer functions - namely, choosing the highest order of the numerator and denominator from the transfer function (shown in Eq. (1.7)). Then apply complex non-linear

complex fit methods for the data to get the system parameters $\hat{\theta}$ of the transfer function for each frequency response, which could also be achieved by a MATLAB ${ }^{\circledR}$ built in function, such as invfreqs(). However, the function in 
MATLAB, nearly a black-box for users, may converge to different local minimum solutions, hard for fine tuning process. If any appropriate optimization objective function with a good initial value is chosen, the algorithm can help us to find a solution within one group of local minimum. Here the magnitude of complex difference error as an objective function in the algorithm will be better to optimize.

$$
|\operatorname{FFTdata}(\omega)-[G(\hat{\theta})](\omega \cdot j)|
$$

Since all values for convergence are assuming to be the neighborhood of initial value within one group, it is reasonable to set one initial value for the algorithm to begin.

This vector form fitting problem can be solved by setting an appropriate norm function, the fitting function can be denoted as follows,

$$
h(\{x\})=\left\{h_{1}\left(x_{1}\right), h_{2}\left(x_{2}\right), \ldots, h_{n}\left(x_{n}\right)\right\}
$$

Where $h(\{x\})$ is the vector form fitting function; $\{x\}$ is the variable in vector form (i.e. $\{x\}=\left\{x_{1}, x_{2}, \ldots, x_{n}\right\}$ ); assuming that $\{\hat{X}\}$ is the data need to be fitted by fitting function.

And the objective function usually can be denoted as a norm function:

$$
\begin{aligned}
H(x, \hat{X}) & =\left\|h(\{x\})-\left\{\hat{X}_{i}\right\}\right\|_{p} \\
& =\left(\sum_{i}\left(h_{i}\left(x_{i}\right)-\hat{X}_{i}\right)^{p}\right)^{\frac{1}{p}}
\end{aligned}
$$


Where $H(x, \hat{X})$ is the objective function in the optimization process, keeping it to the minimum in order that the fitting function $h(\{x\})$ will approximately fit to the data $\{\hat{X}\}$.

The norm function $\|\square\|_{p}$ is the $p$ norm, which could be adopted into higher dimension fitting problem. The non-linear complex function fit can be simply considered as a fitting process on real number and imaginary number two dimensional problem, namely:

$$
H=\|\{\operatorname{Re}(G), \operatorname{Im}(G)\}-\{\hat{X}\}\|_{p}
$$

Where $\{\hat{X}\}=\{\operatorname{Re}(\hat{X}), \operatorname{Im}(\hat{X})\}$.

If the norm is 2-norm, $H$ is equivalent to the magnitude of complex number.

$$
H=|G-\hat{X}|
$$

Where $\hat{X}$ is a complex number (here $\hat{X}=\operatorname{Re}(\hat{X})+\operatorname{Im}(\hat{X}) \cdot j$; where $j$ is unit imaginary number).

The following procedures in this subsection follow the techniques first developed by $[3,5]$. Define the set of frequency response transfer functions for samples $R[3,5]$ as follows:

$$
R:=\{[G(\hat{\theta})](s) ; \hat{\theta} \in \hat{\Theta}\}
$$




$$
\begin{aligned}
& {[G(\hat{\theta})](s)=\frac{\sum_{i=0}^{m} b_{i+1} \cdot s^{m-i}}{\sum_{j=0}^{n} a_{j+1} \cdot s^{n-j}}} \\
& \hat{\theta}_{0}=\left\{b_{1}, \ldots, b_{m+1}, a_{1}, \ldots, a_{n+1}\right\}
\end{aligned}
$$

And system parameters can be further reduced since we can force one parameter to be a constant just by dividing a number proportional to this parameter on both the numerator and denominator. In this article, the parameter $b_{1}$ is forced to be 1 . Then the system parameters could be,

$$
\begin{aligned}
\hat{\theta}_{k} & =\frac{1}{b_{k, 1}} \cdot\left\{b_{k, 2}, \ldots, b_{k, m+1}, a_{k, 1}, \ldots, a_{k, n+1}\right\} \\
& =\left\{\tilde{b}_{k, 2}, \ldots, \tilde{b}_{k, m+1}, \tilde{a}_{k, 1}, \ldots, \tilde{a}_{k, n+1}\right\}
\end{aligned}
$$

Then, a parameter set can be built,

$$
\hat{\Theta}:=\left\{\hat{\theta}_{k} \in \mathbb{R}^{q} ; k=1, \ldots, \kappa\right\}
$$

where $k$ is the index of sample sets from $\hat{\Theta}, \kappa$ is the number of $\hat{\theta}$ in $\hat{\Theta}$, and $q$ is the dimension of system parameters. The system parameter set $\hat{\Theta}$ can be approximated by defining a set $\Theta$ as,

$$
\Theta:=\left\{\theta_{k}:=f(\lambda) ; k=1, \ldots, \kappa\right\}
$$

where $f(\lambda)$ can be defined as.

$$
\left\{\begin{array}{l}
f(\lambda)=[C]\{\lambda\} \\
0 \leq \lambda_{k} \leq 1
\end{array}\right.
$$


This type of uncertainty model fits only the situation when the reduced dimension geometry is a topological homomorphism with an open ball (or open disk); however, other geometry with different topological structure needs further space transformation projecting on Cartesian space. In this paper, we only consider the situation without further space transformation.

From Eq. (1.12), the matrix $[C]$ is the uncertainty parameters, and vector $\{\lambda\}$ is a vector of uncertainty variables, but with some certain rules. For instance, if the highest order of uncertainty is 3 , and the number of uncertainty variables is 2 , then the uncertainty variable vector will be following equation,

$$
\{\lambda\}=\{\underbrace{1}_{v_{0}(\lambda)^{T}}, \underbrace{\lambda_{1}, \lambda_{2}}_{v_{1}(\lambda)^{T}}, \underbrace{\lambda_{1} \lambda_{2}, \lambda_{1}{ }^{2}, \lambda_{2}^{2}}_{v_{2}(\lambda)^{T}}, \underbrace{\lambda_{1} \lambda_{2}^{2}, \lambda_{1}^{2} \lambda_{2}, \lambda_{1}^{3}, \lambda_{2}^{3}}_{v_{3}(\lambda)^{T}}\}
$$

where $v_{i}(\lambda)$ defines the sub-vector $\lambda$ with $i^{\text {th }}$ order with a highest summation of the order of variables.

Variable vector $\{\lambda\}$ defined in this form contains an advantage, i.e. when doing a linear space transformation, the formula could be denoted by itself by introducing a transformation matrix $\left[T_{\lambda}\right]$.

$$
\{a \cdot \lambda+b\}=\left[T_{\lambda}\right]\{\lambda\}
$$

Such as the condition of $2^{\text {nd }}$ order, 2 variables. 


$$
\begin{aligned}
\{a \cdot \lambda+b\} & =\left\{\begin{array}{l}
1 \\
a \cdot \lambda_{1}+b \\
a \cdot \lambda_{2}+b \\
\left(a \cdot \lambda_{1}+b\right)\left(a \cdot \lambda_{2}+b\right) \\
\left(a \cdot \lambda_{1}+b\right)^{2} \\
\left(a \cdot \lambda_{2}+b\right)^{2}
\end{array}\right\}=\left\{\begin{array}{l}
1 \\
a \cdot \lambda_{1}+b \\
a \cdot \lambda_{2}+b \\
b^{2}+a \cdot b \lambda_{1}+a \cdot b \lambda_{2}+a^{2} \lambda_{1} \lambda_{2} \\
b^{2}+2 a \cdot b \lambda_{1}+a^{2} \lambda_{1}^{2} \\
b^{2}+2 a \cdot b \lambda_{2}+a^{2} \lambda_{2}^{2}
\end{array}\right\} \\
& =\left[\begin{array}{cccccc}
1 & 0 & 0 & 0 & 0 & 0 \\
b & a & 0 & 0 & 0 & 0 \\
b & 0 & a & 0 & 0 & 0 \\
b^{2} & a \cdot b & a \cdot b & a^{2} & 0 & 0 \\
b^{2} & 2 a \cdot b & 0 & 0 & a^{2} & 0 \\
b^{2} & 0 & 2 a \cdot b & 0 & 0 & a^{2}
\end{array}\right]\left\{\begin{array}{l}
1 \\
\lambda_{1} \\
\lambda_{2} \\
\lambda_{1} \lambda_{2} \\
\lambda_{1}{ }^{2} \\
\lambda_{2}{ }^{2}
\end{array}\right\}=\left[T_{\lambda}\right] \cdot\{\lambda\}
\end{aligned}
$$

Since this problem is based on a numerical method, the normalization of the data is necessary to ensure the fidelity of the information. Here, the system parameter $\hat{\theta}_{k}$ has been normalized to be $\hat{\theta}_{k}^{\prime} \in(-1,1)^{q}$.

$$
\left\{\hat{\theta}_{k}^{\prime}\right\}=\frac{2\left(\left\{\hat{\theta}_{k}\right\}-\min \left(\left\{\hat{\theta}_{k}\right\}\right)\right)}{\max \left(\left\{\hat{\theta}_{k}\right\}\right)-\min \left(\left\{\hat{\theta}_{k}\right\}\right)}-\{1\}=\left[T_{\hat{\theta}}\right]\left\{\hat{\theta}_{k}\right\}
$$

The inverse calculation can be applied on both $\hat{\theta}_{k}$, and $\theta_{k}$ since it can be treated as the same when getting a good approximation.

$$
\begin{aligned}
& \left\{\begin{array}{l}
\left\{\hat{\theta}_{k}\right\}=\left[T_{\hat{\theta}}\right]^{-1}\left\{\hat{\theta}_{k}^{\prime}\right\} \\
\left\{\theta_{k}\right\}=\left[T_{\hat{\theta}}\right]^{-1}\left\{\theta_{k}^{\prime}\right\}
\end{array}\right. \\
& \Rightarrow f\left(\lambda_{k}\right)=\left[T_{\hat{\theta}}\right]^{-1} f^{\prime}\left(\lambda_{k}\right) \\
& {[C]=\left[T_{\hat{\theta}}\right]^{-1}\left[C^{\prime}\right]}
\end{aligned}
$$




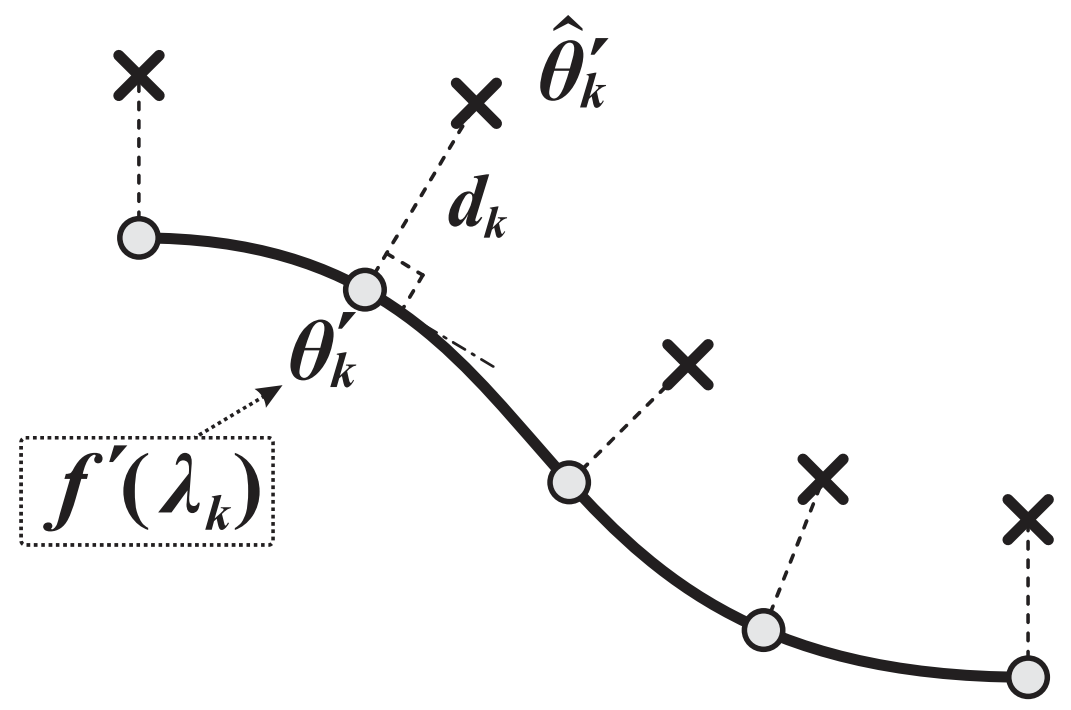

Figure 2.1 Distance $d_{k}$ for a set of samples

The corresponding normalized system parameter in uncertainty model $\theta_{k}^{\prime}$ is a point on the reduced-dimension geometry with the minimum distance $d_{k}$ from the higher dimension point (shown in Figure 2.1), and its distance $d_{k}$ can be defined as[3],

$$
\begin{aligned}
d_{k} & :=\min \left(\left\|\hat{\theta}_{k}^{\prime}-f^{\prime}(\lambda)\right\|\right) \\
& =\sqrt{\min \left(\left\|\hat{\theta}_{k}^{\prime}-f^{\prime}(\lambda)\right\|^{2}\right)}=\left\|\hat{\theta}_{k}^{\prime}-\theta_{k}^{\prime}\right\|
\end{aligned}
$$

Where the norm function we are defined as the 2 norm:

$$
\|\{x\}\|_{2}=\sqrt{\sum_{i} x_{i}^{2}}
$$

The $\theta_{k}^{\prime}$ could be solved by numerical optimization method. In other words, since $\left\|\hat{\theta}_{k}^{\prime}-f(\lambda)\right\|^{2}$ is also a non-linear polynomial with variables $\lambda$, various 
numerical methods could be applied to find its minimum. Then, each value of $\lambda_{k}$ and its corresponding value $\theta_{k}^{\prime}$ and $d_{k}$ under each condition can also be found. In this article, the unbounded optimization method is applied to do the minimization problem due to its high efficiency comparing with bounded algorithms. Then, all values can be converted back to bounded conditions by the same "normalization method"; which means that, the optimization program will automatically search $\left[\bar{C}^{\prime}\right]$ and $\{\bar{\lambda}\}$, and the domain of $\{\bar{\lambda}\}$ can be converted back into $\lambda \in(0,1)$ by linear transformation shown as Eq. (1.14). This transformation will be calculated on the final step of the GA as,

$$
\begin{aligned}
& \{\lambda\}=\frac{\{\bar{\lambda}\}-\min (\{\bar{\lambda}\})}{\max (\{\bar{\lambda}\})-\min (\{\bar{\lambda}\})}=\left[T_{\lambda}\right] \cdot\{\bar{\lambda}\} \\
& \Rightarrow f^{\prime}(\lambda)=\left[C^{\prime}\right]\{\lambda\}=\underbrace{[C] \cdot\left[T_{\lambda}\right]^{-1}}_{\left[\bar{C}^{\prime}\right]}\{\bar{\lambda}\} \\
& \Rightarrow\left[C^{\prime}\right]=\left[\bar{C}^{\prime}\right]\left[T_{\lambda}\right]^{-1}
\end{aligned}
$$

where $\left[T_{\lambda}\right]$ is the transformation matrix for variable $\lambda$.

In order to make sure the geometry can largely pass all points, we would try to make sure summation of all distance $D$ to be the minimum, where

$$
D:=\sum_{k=1}^{\kappa} d_{k}
$$

In this article, the Genetic Algorithm is adopted to improve the result gradually. 


\section{CHAPTER 3: GENETIC ALGORITHM OPTIMIZATION OF UNCERTAINTY MODEL COEFFICIENTS}

\subsection{Why Using GA}

Although other researchers have already developed several methods to find system parameters described in the previous section $[3,5]$, the general algorithm can be problematic in solving non-convex problems. However, previous approaches to curve or multi-dimensional polynomial fitting using GA[7, 8]

dealing with non-convex low dimensional geometry approximation gives us the idea that parameter optimization by GA can be adopted in this parameterization problem, a good solution can be found by running the GA several times, allowing the solution to further reduce the cost function.

\subsection{Overview of GA}

Genetic Algorithm (GA), one of an evolutionary artificial intelligence method, is a search heuristic that mimics the natural selection process and generate useful solutions to optimization and search problems, which is strong in solving the nonlinear, non-convex as well as huge and complicated optimization problems. The main technique for this algorithm is inheritance, mutation, selection and crossover, and iteratively pick out a best solution among various solutions. Nevertheless, basic GA algorithm cannot suitable for every case, therefore a fine tuning on the algorithm for a specific case is needed. The procedure of tuning process is shown below. 


\subsection{Procedure of GA Optimization}

Our approach of optimization the uncertainty model parameterization with a GA is given in the following steps.

STEP 1. Initialize Genes and Population

The system parameters could be encoded by converting to binary with $l$ binary digits.

$$
\begin{aligned}
c_{i j} & =\operatorname{to}_{10}\left(\left\{c_{i j}\right\}\right) \\
& =\operatorname{to}_{10}\left(\left\{c_{i j(1)}, \ldots, c_{i j(n)}, \ldots, c_{i j(l)}\right\}\right)
\end{aligned}
$$

where $c_{i j(n)}$ represents each component of matrix $\left[\bar{C}^{\prime}\right]$ at the $n^{\text {th }}$ binary digit; and $c_{i j(n)}$ is either 0 or $1 . t o_{10}(x)$ is the function converting the binary list to the number we used.

An encoding function can be defined as

$$
t_{10}(\{x\})=\varphi \cdot \frac{\sum_{i=1}^{l}\left(2^{l-i} \cdot x_{i}\right)-\sum_{i=1}^{l}\left(2^{l-i-1}\right)}{\sum_{i=1}^{l}\left(2^{l-i-1}\right)}
$$

where $\varphi$ is the maximum number of parameters. Then, randomly generate

$2 \eta$ matrix $\left[\bar{C}^{\prime}\right]$ as the initial population pool, the information kept in binary form; population number is $2 \eta$, and $p$ is the index of gene in the population pool. 


\section{STEP 2. Selecting}

By using Eq.(1.18) and Eq. (1.21), we can generate series of summation of distance errors, denoted as $D_{p}$. Before introducing the probability parameter, the untwisted coefficient and converge coefficient should be defined.

The normalized function will be defined as

$$
\mathrm{N}(\{x\})=\frac{\{x\}}{\max (\{x\})}
$$

Since the coefficient of polynomial with a higher order of variables will contribute to more ripples on the geometry, the ripple coefficient $\Lambda$ will be defined to control the twisted effect on the geometry.

$$
\{\Lambda\}=\left.\{\lambda\}\right|_{\lambda_{1, \ldots i}=\gamma}
$$

Since the geometry has been normalized by series of transformations, the effect of ripple coefficient acting on each dimension would be the same; thus choosing only one constant $\gamma$ to define the ripple coefficient $\{\Lambda\}$ is enough. Unfortunately, mathematical proof can never be found to prove the untwisted effect of the geometry; however, it works pretty well in the majority cases.

The untwisted coefficient $t_{p}$ can be defined by assuming that the smaller value of normalization of the summation of all numbers in matrix $\left[\bar{C}^{\prime}\right]$ times ripple coefficient $\{\Lambda\}$.

$$
t_{p}=\mathrm{N}\left(\left\{\sum([C] \cdot\{\Lambda\})\right\}_{p}\right)
$$


Thus, the probability function can be defined as.

$$
\Pi_{p}=\beta^{-\left(\alpha \cdot \frac{D_{p}}{\kappa} \cdot \mathrm{N}\left(t_{p}-\min \left(\left\{t_{p}\right\}\right)+u\right) \cdot \mathrm{N}\left(D_{p}-v \cdot \min \left(\left\{D_{p}\right\}\right)\right)\right)}
$$

The term $\mathrm{N}\left(t_{p}-\min \left(\left\{t_{p}\right\}\right)+u\right)$ includes addition of the obvious coefficient $u$ which can modify the effect of untwisted coefficient to be one probability influence factor. The term $\mathrm{N}\left(D_{p}-v \cdot \min \left(\left\{D_{p}\right\}\right)\right)$ includes the converge coefficient $v$ which can control the weight of effect on the convergence of the summation of distance $D_{p}$. Constant $\beta$ is the parameter to convert the probability function into the power style function. The constant $\alpha$ is the shape correction coefficient for the power function. In our program, each of these will be adjusted in a real time with the iteration of calculation - and the values will be $\alpha=50$, $\beta=3, \varphi=1.5, \gamma \in(1,5), u \in(0.2,250), v \in(0,1)$.

Then, randomly select $\eta$ pairs of "parents" from the pool with the probability $\left\{\Pi_{p}\right\}$ by the roulette wheel method,

In order to improve the solution, a technique can be used to make sure the population always contains the best solution ever had. A technique introducing "schemata" can be used that is defined as randomly dropping one of the population genes and adding the best solution back to the pool in each iteration. Then, the parents can be denoted as

$$
P_{i}=\left\{P_{i, 1}, P_{i, 2}\right\}
$$


STEP 3. Crossover and Mutation

The offspring, $O_{i}$ can be produced by crossover and mutation methods from its parents $P_{i}$. The procedures are shown in Figure 3.1: each offspring will be treated independently by randomly selecting the gene from both parents with $50 \%$ probability.

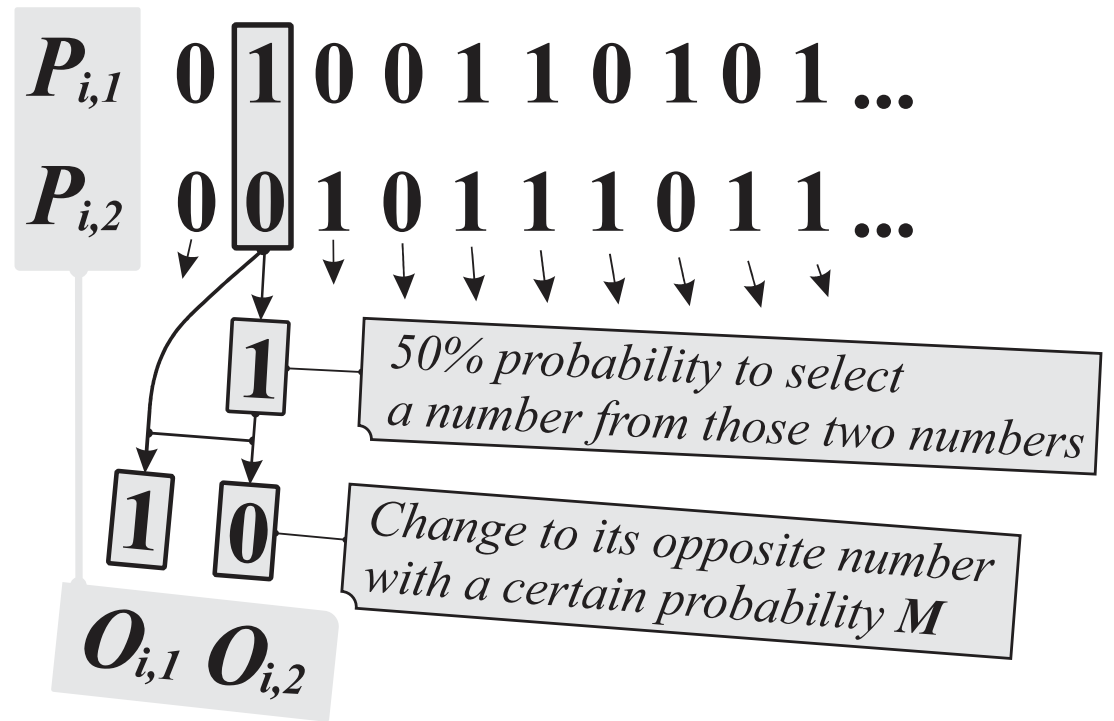

Figure 3.1 Crossover and Mutation

Then, the mutation algorithm will be applied on each gene of offspring with a certain probability.

Better convergence could be achieved by the dynamically changing the mutation rate $M$ dropped with the convergence of the minimum sum of distance from all populations. Then, define $\mu$ as the mutation coefficient,

$$
M=\mu \min \left(\left\{D_{p}\right\}\right)
$$




\section{STEP 4. Iteration}

Return to STEP 2 for iteration, and watch for the convergence of the algorithm by considering three values which could indicate the convergence of the algorithm.

1. The average value of summation of distance error: mean $\left(\left\{D_{p}\right\}\right)$;

2. The minimum value of the summation of distance error: $\min \left(\left\{D_{p}\right\}\right)$, which is the most significant parameter;

3. The average value of probability parameter: mean $\left(\left\{\Pi_{p}\right\}\right)$;

No specific requirement for convergence is known for these parameters in 1-3 above. These parameters can be used by trial and error to determine when the algorithm has converged on a good solution by also viewing the solution graphically.

STEP 5. Getting the Result

Since the uncertainty parameter and uncertainty variables are under two types of normalized transformation, they should be converted back to get the final result for the uncertainty model. From Eq. (1.17), (1.20).

$$
[C]=\left[T_{\hat{\theta}}\right]^{-1}\left[C^{\prime}\right]=\left[T_{\hat{\theta}}\right]^{-1}\left[\bar{C}^{\prime}\right]\left[T_{\lambda}\right]^{-1}
$$

\subsection{Test Uncertainty Model}

Here we test a simple example of a surface in 3 dimensional space. The point cloud is part of function $\sin (x \cdot y)$, where $x, y \in(-1.5,1.5)$. Then adopt 
uncertainty model to fit this surface with 2 variables and the highest order at 3 . The result:

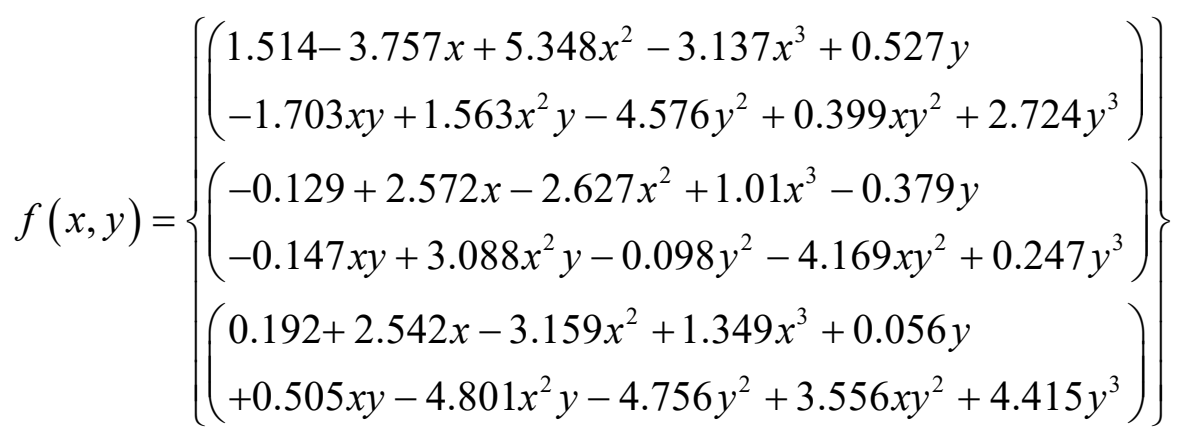

The fitting plot is shown in Figure 3.2 and its corresponding values for uncertainty variable are shown in Figure 3.3:

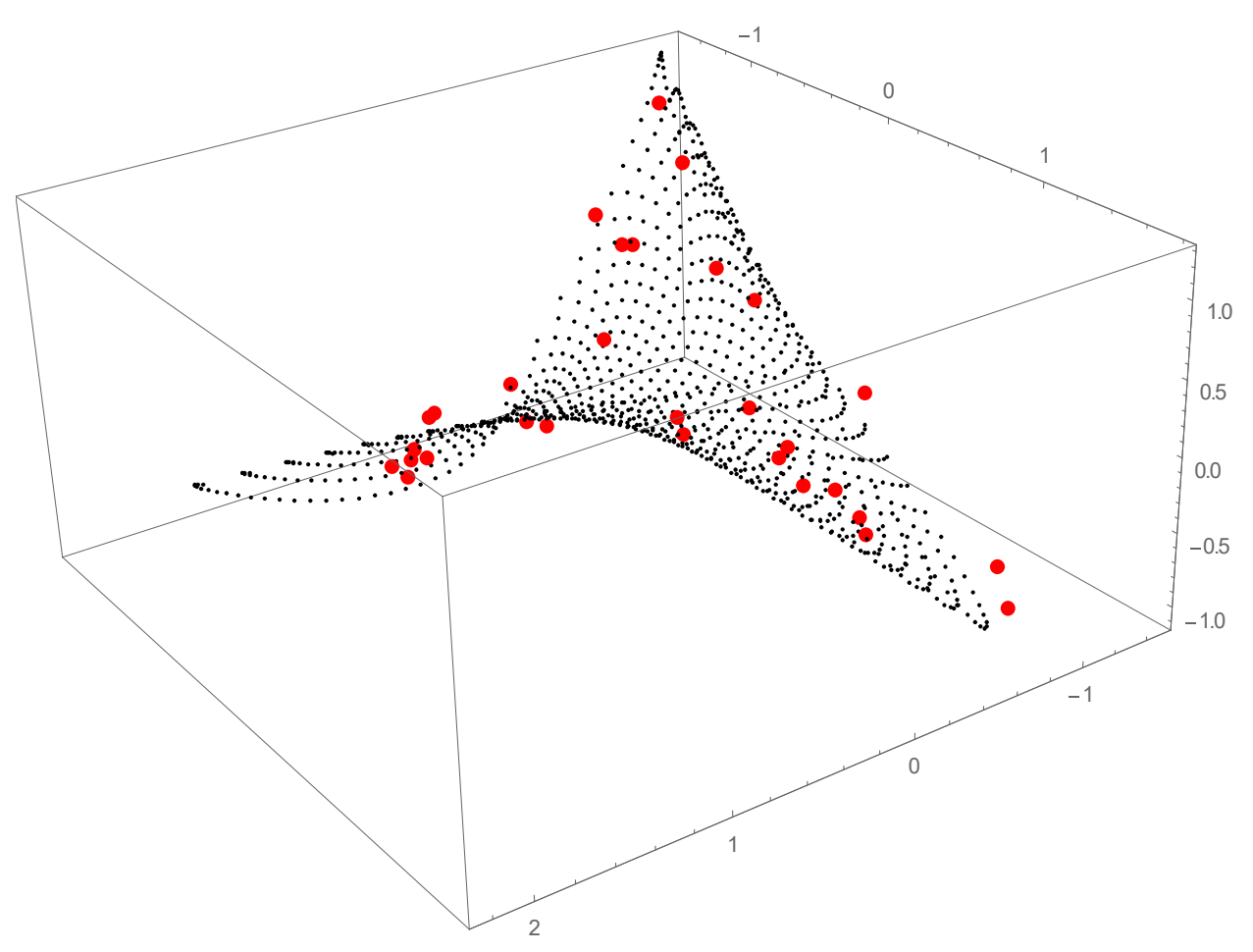

Figure 3.2 The Function Fit for $\sin (x \cdot y)$ 


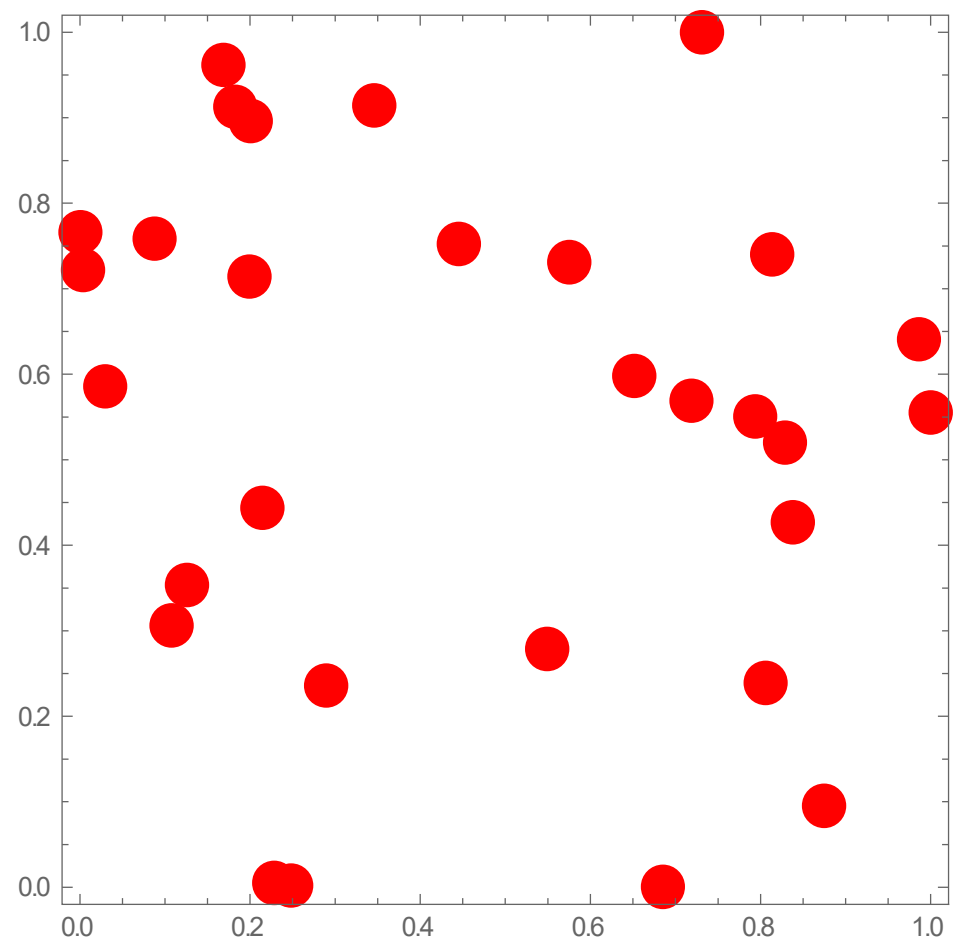

Figure 3.3 Value of Uncertainty Variables $\lambda_{1}$ and $\lambda_{2}$

We can see that the points are on the surface, which validate the effectiveness of the algorithm of our uncertainty model. 


\section{CHAPTER 4: PRACTICAL EXAMPLE - ELECTRO- HYDRAULIC VALVE}

\subsection{Example Background}

To illustrate the advantages in uncertainty modeling that can be used in a robust stability test, let us go through an example from [2,9]. The example application is of an electrohydraulic valve that has two stages, an electrohydraulic pressure control pilot stage, and a larger main stage that can control large hydraulic fluid flows. The pilot stage is operated directly by a solenoid and is used to control pressure that actuates the main stage of the valve. Pressure on one side of the main stage causes it to shift and compress a stiff spring, which balances with the pressure force. The positioning of the main valve is then proportional to the signal controlling the pilot stage. The input signal is a voltage as a chirp signal sent to an amplifier that then supplies a proportional current to the solenoid. The output is the position of the main valve. There were a total of 30 replications of this valve setup that were tested to represent the uncertainty in a mass produced valve from a manufacturer. More details of the experimental setup and the frequency response data obtained can be found in the cited works.

\subsection{Close Loop System Analysis}

The raw data are from the experiments utilizing 30 electrohydraulic valves. The transfer function of the frequency response given voltage inputs with position output can be obtained by taking the fast Fourier transformation (FFT) of both the 
input and output (control voltage and position respectively), and taking the ratio of these. The input of chirp signal is shown below:
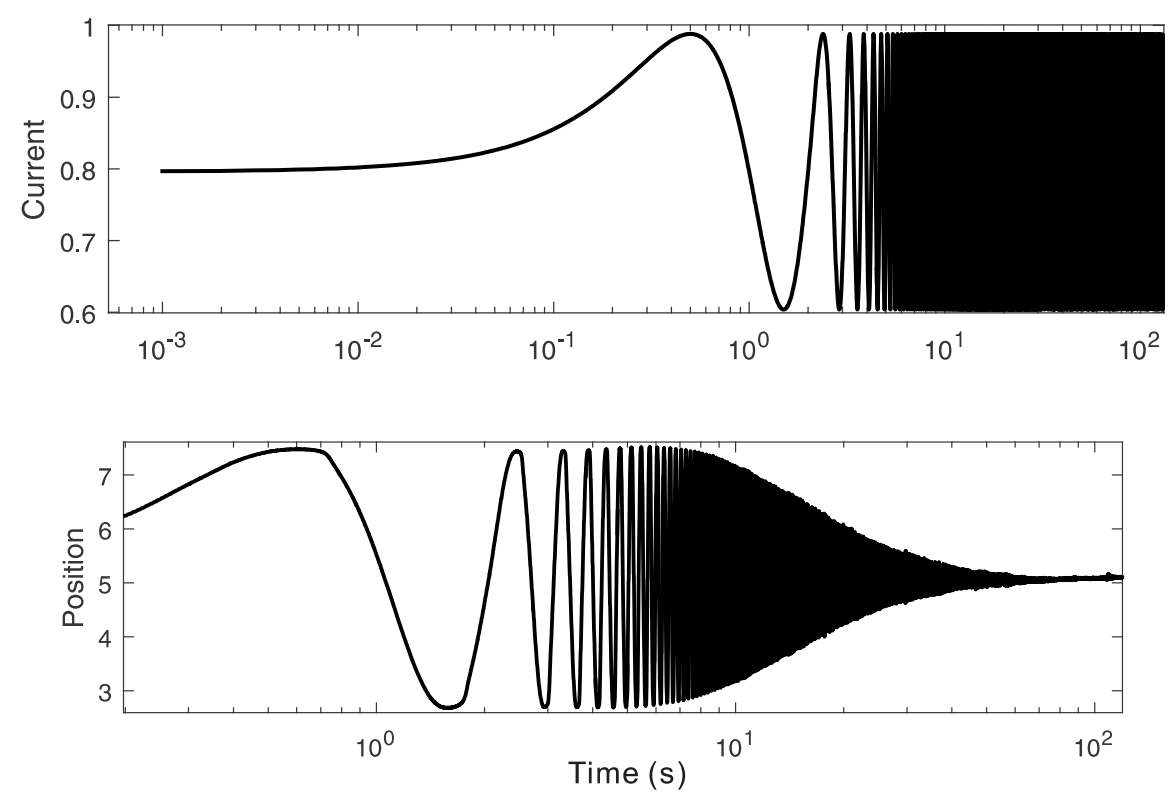

Figure 4.1 The Input Chirp Signal and Corresponding Response in Time Domain

Then, the frequency response for 30 is presented below:
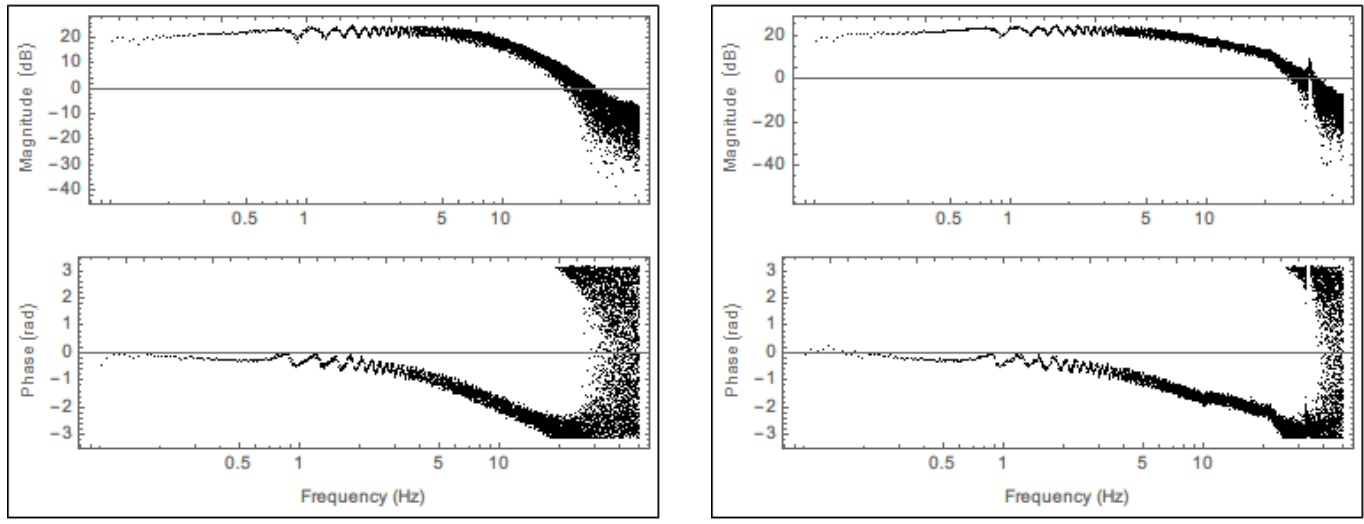

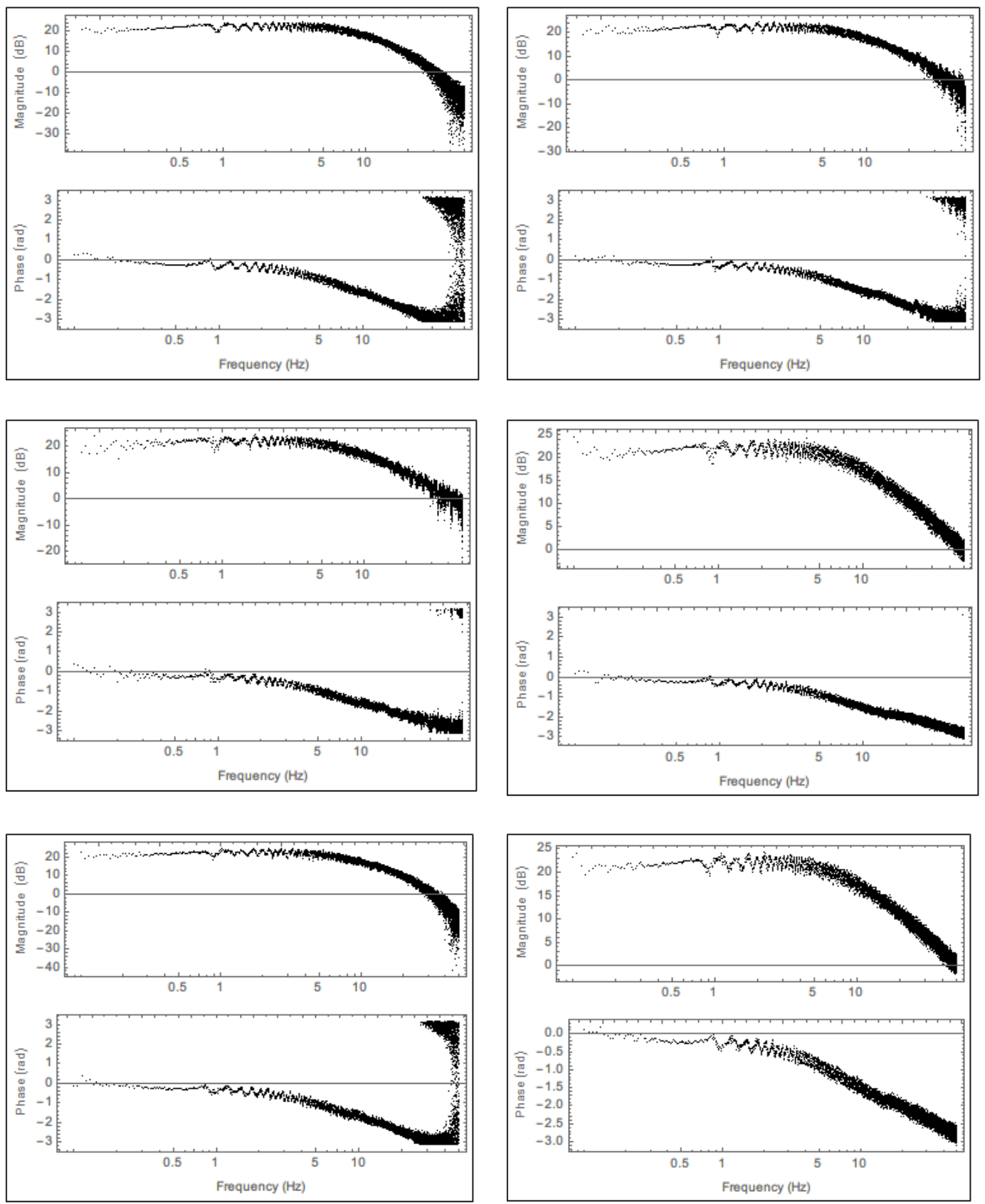

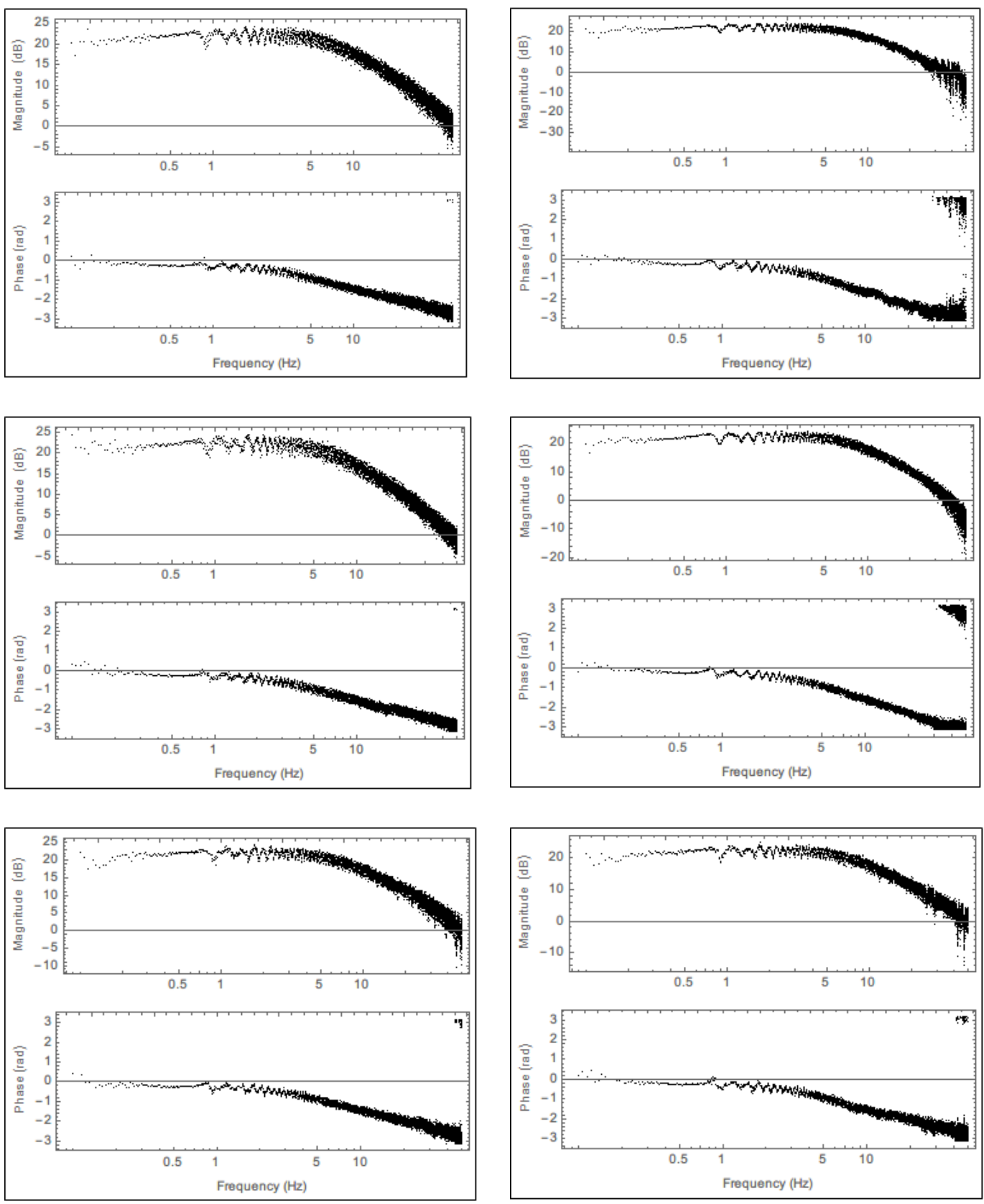

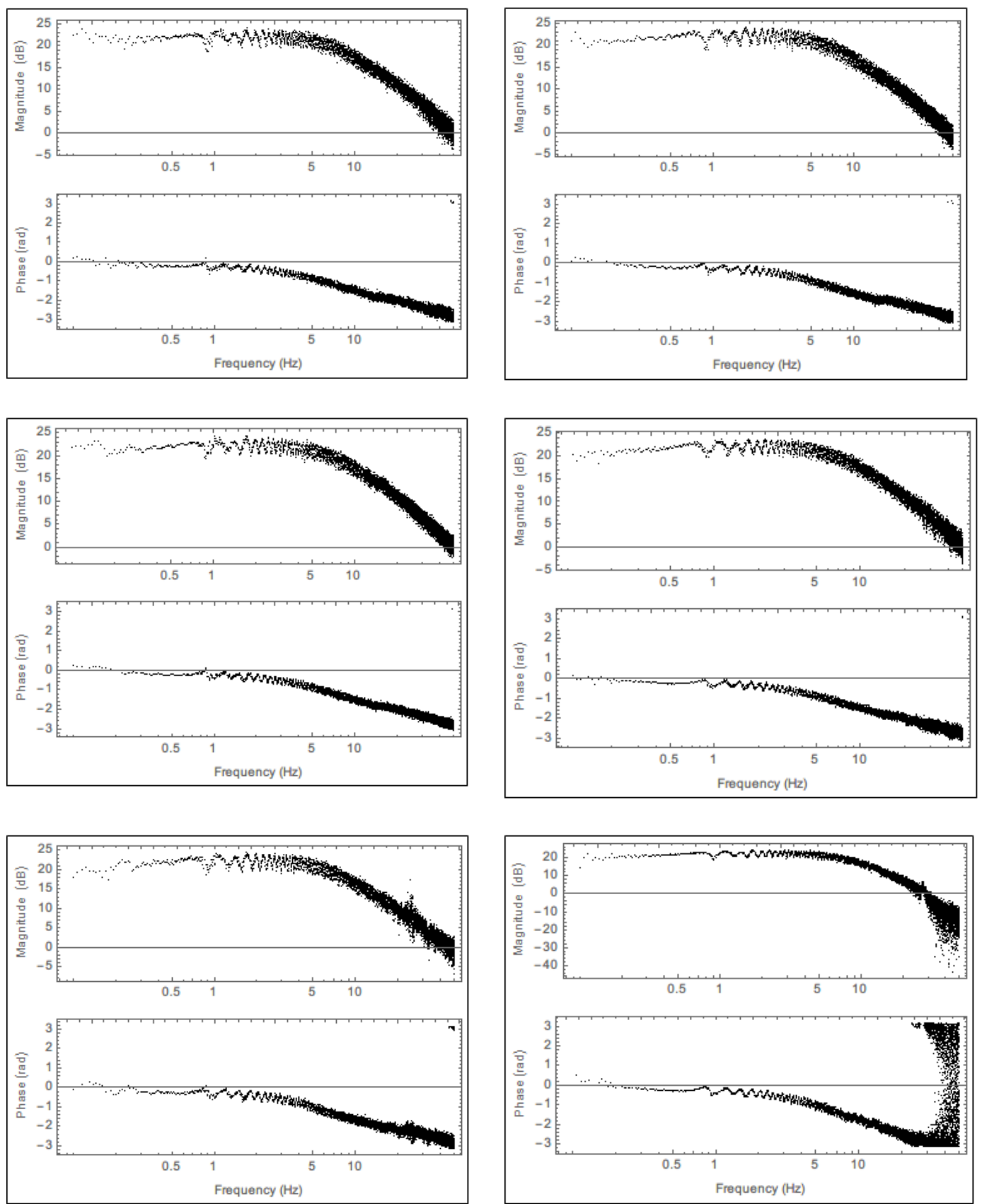

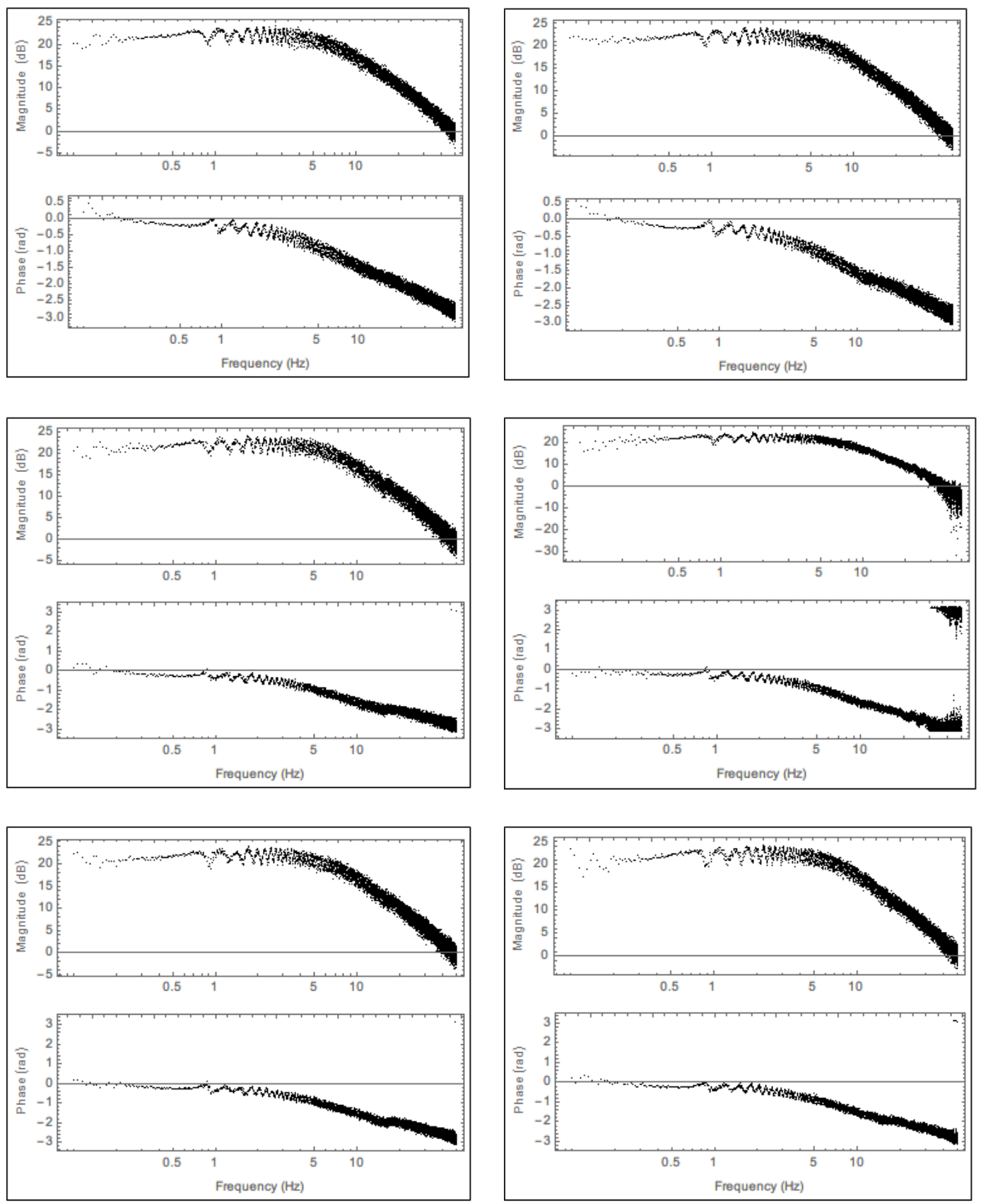

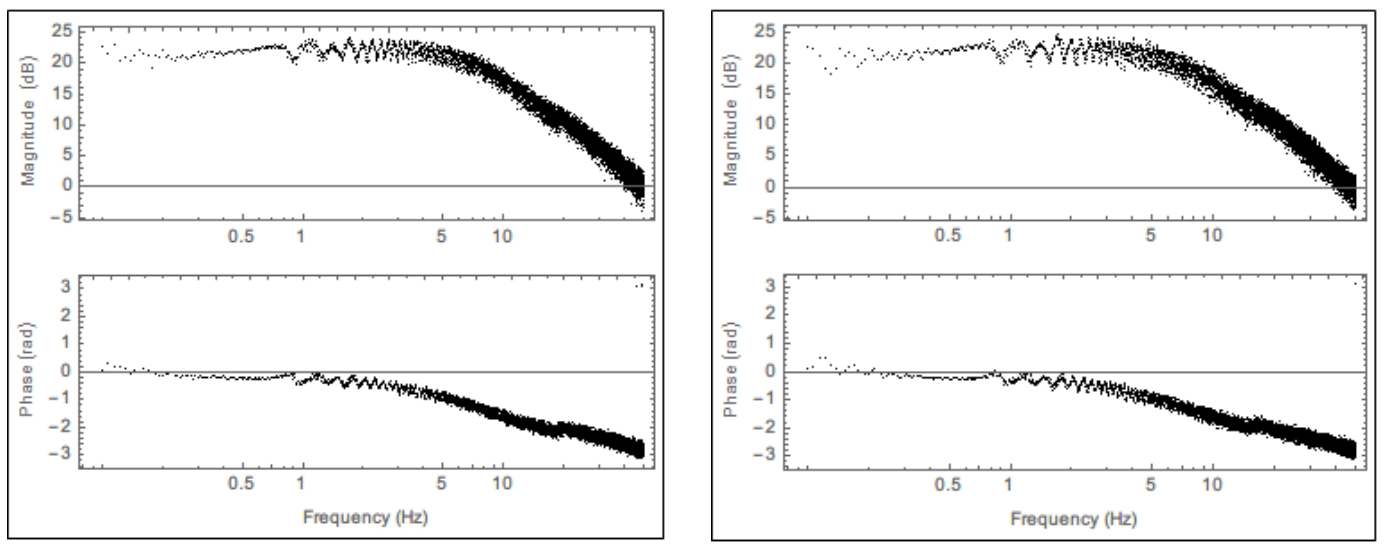

Figure 4.2 List of Frequency Response of 30 Replicative Valves

Then, we use the MATLAB function "infreqs()", a command that using the complex nonlinear optimization method, to fit and generate the system parameters $\theta$ 's for a transfer function to each frequency response of the data set. The frequency response of an example of one valve is given in Figure 4.3 Note that the fitting process is only applied to data in the frequency range shown by the solid line. The order of transfer function can be estimated by numerous trial and error, and can be governed by the structure below in Eq. (3.1), where $\tilde{b}_{2}, \tilde{a}_{1}, \tilde{a}_{2}$ and $\tilde{a}_{3}$ are some specific value in this structure:

$$
[G(\hat{\theta})](s)=\frac{s+\tilde{b}_{2}}{\tilde{a}_{1} s^{2}+\tilde{a}_{2} s+\tilde{a}_{3}} ; \hat{\theta}:=\left\{\tilde{b}_{2}, \tilde{a}_{1}, \tilde{a}_{2}, \tilde{a}_{3}\right\}
$$



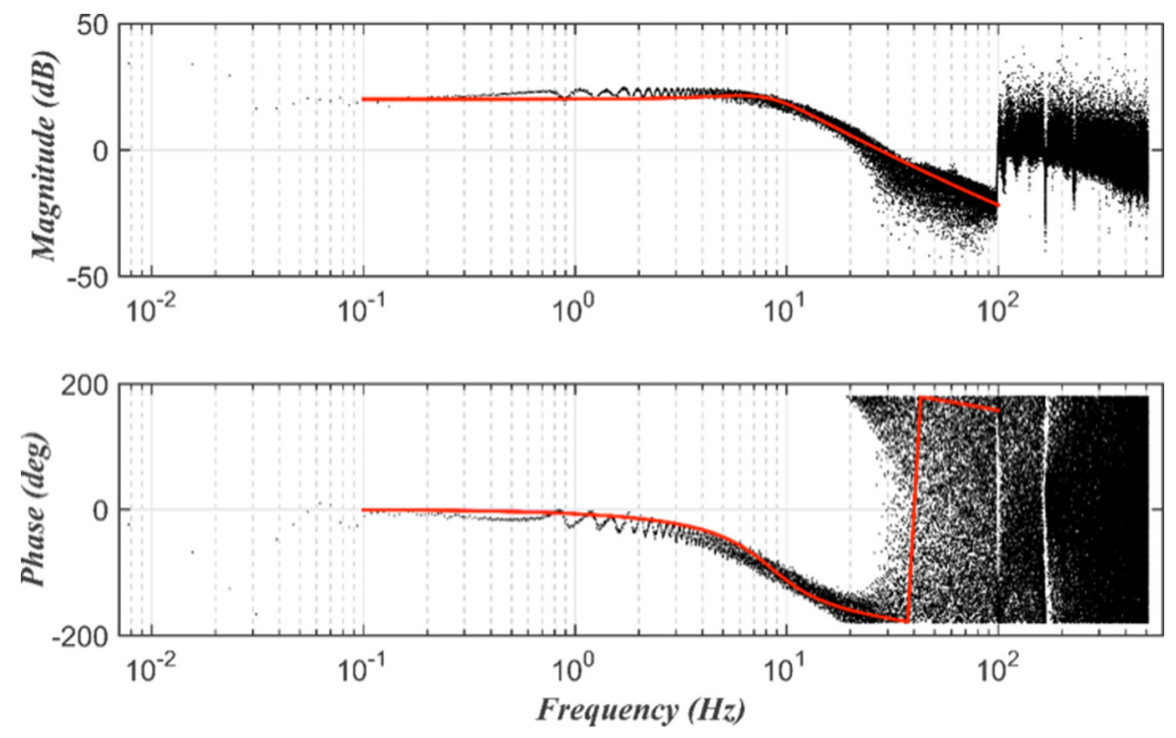

Figure 4.3 Frequency response fit (solid line) for transfer function for example data (dots) from one valve

The dynamic response of each of the 30 valves can be treated equivalently with these system parameter set $\hat{\Theta}$ from the transfer function $G(s)$. By plotting point cloud, we can first determine frequency response is valid below $30 \mathrm{~Hz}$, and then decide that the system parameters (4 dimensional vector sets) could pass through a one dimensional line ( $2^{\text {nd }}$ order function in $\lambda$ in our program) whose mathematical expression could be obtained by uncertainty model with the GA method described earlier. And the results are shown in Eq. (3.2). The fit function is shown in Figure 4.4 and the range of values of $\lambda$ are shown in Figure 4.5.

$$
\begin{aligned}
f(\lambda) & =[C]\{\lambda\} \\
& =\left[\begin{array}{ccc}
-387.59 & -170 & 1617.53 \\
-0.44 & 0.41 & 0.78 \\
-4.74 & -1.97 & 19.73 \\
-29.05 & -25.52 & 138.37
\end{array}\right]\left\{\begin{array}{l}
1 \\
\lambda \\
\lambda^{2}
\end{array}\right\}
\end{aligned}
$$




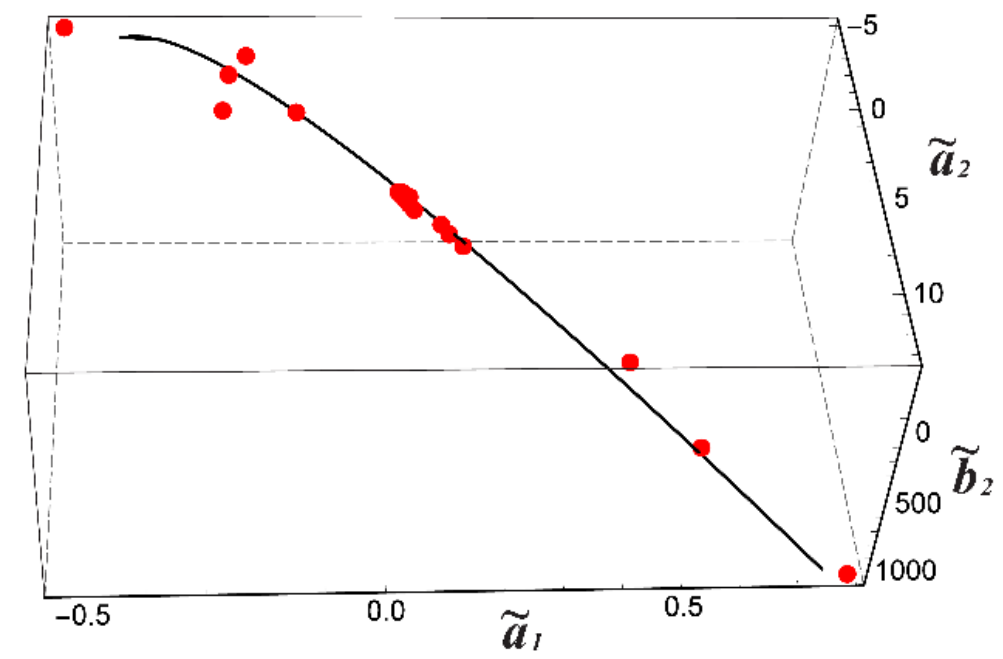

Figure 4.4 GA fit for point cloud in 3-Dimensional View

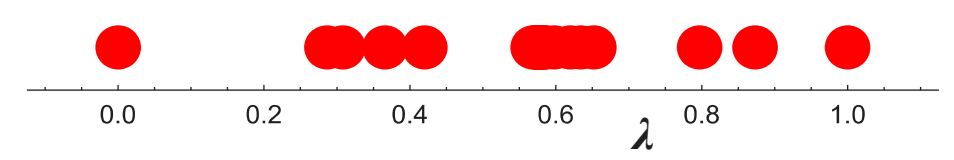

Figure 4.5 Value of $\lambda$ on number-line

However, there exists a singular region which the shape of transfer function would change sharply when $\lambda \in(0.421,0.573)$, and therefore can be deleted. This suggests that there are at least two groups of plants that have some significantly different characteristics.

Now, we will consider a simple proportional feedback control system for the valve. A block diagram of the control system with uncertainty parameterized by $\lambda$ is shown in Figure 4.6. 


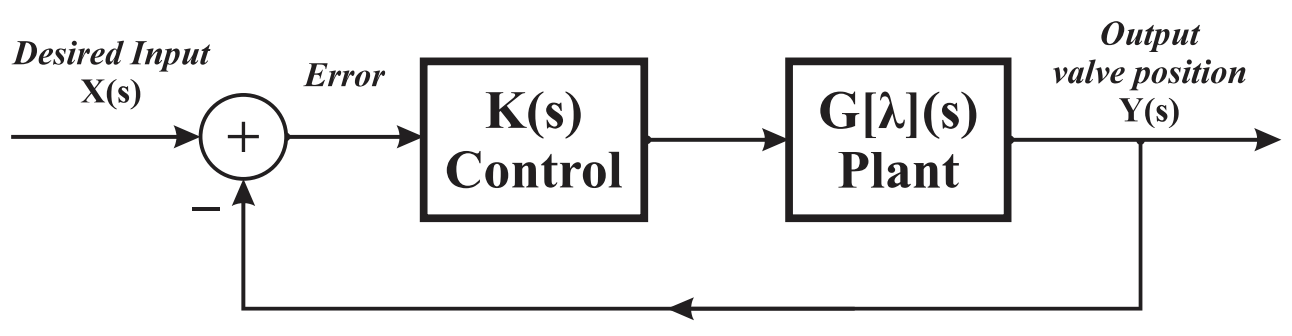

Figure 4.6 Analysis of Feedback Control for Uncertainty Model

Here, we assume that $K(s)$ is a constant gain, $K$. The robustness of the system can be estimated by testing the roots of the denominator of the transfer function. This can be done through the use of a root locus for the denominator of the closed loop system, $G K /(1+G K)$. A root locus can be created for all of the range of the uncertainty variable, $\lambda$. For each value of lambda, a root locus can also be created for the gain $K$. For these two variables, multiple root loci could be obtained (See Figure 4.7).

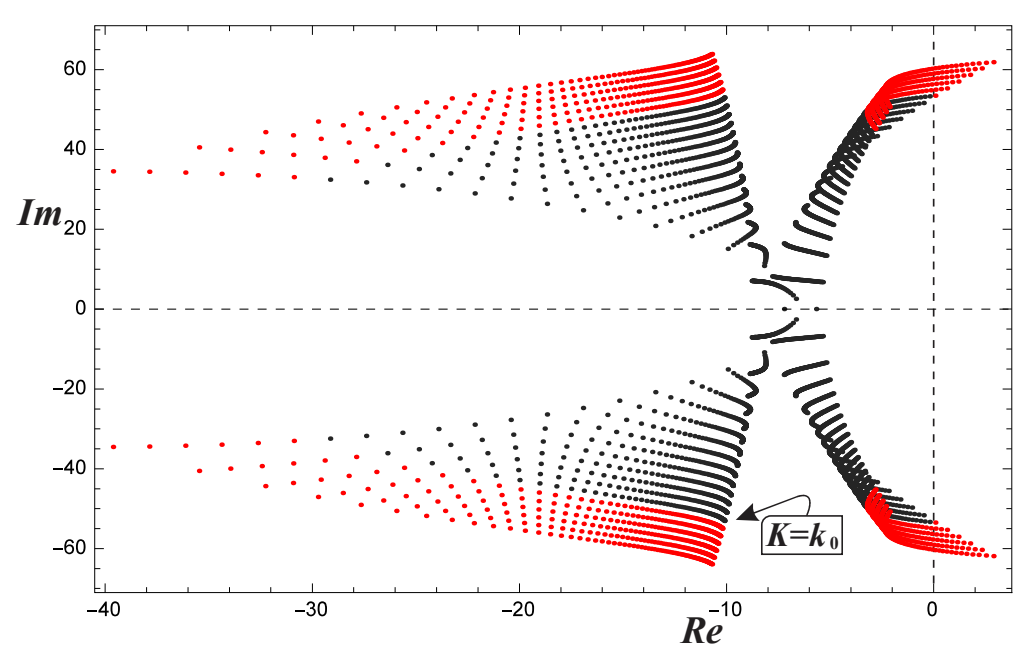

Figure 4.7 The Closed-loop Poles for uncertainty model

Assume that $K=k_{0}$ is the critical condition of the root locus as the poles move to the right half of the complex plane. The edge of Figure 4.7 shows that the 
root loci will reach the right half plane when the control gain is at its critical value $k_{0}=2.02$. The gain, $K$, could span all feasible regions shown in Figure 4.8.

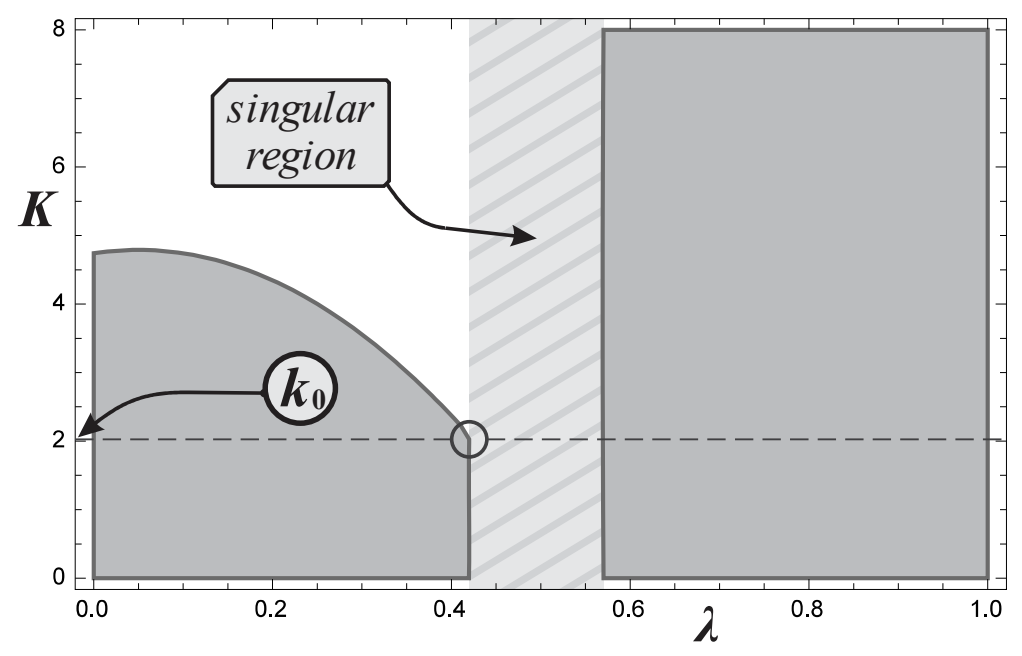

Figure 4.8 Feasible region for $K$ in a stable close-loop controller

Figure 4.8 could also be drawn by using Routh's method stability inequalities. Figure 4.8 shows when $K<k_{0}$, the robustness can be guaranteed. However, small values of $K$ will result in diminished effectiveness with large steady state error. Thus, picking out an appropriate $K$ is a crucial tradeoff between performance and the stability of the system.

\subsection{Refined Model}

Due to the fact when using MATLAB command infreqs(), the optimization algorithm converged to several different local minimum groups of solutions that existing gaps among where the corresponding transfer function cannot smoothly behaved. However, after setting an appropriate initial value for the algorithm, the algorithm could converge correctly to one solution group. Since this optimization algorithm is based on the nonlinear complex function fit for the FFT data 
(complex number), to select an appropriate objective function for optimization is needed, which has been discussed in Eq. (1.1).

Then redo the transfer function fit for 30 valves with one initial value to be selected as one group of value among 30 cases under the same transfer function structure as Eq. (3.1) using nonlinear complex function fit method, and applied into uncertainty model at 2 variables with the highest order 2 .

Take one case to present its fitting result.
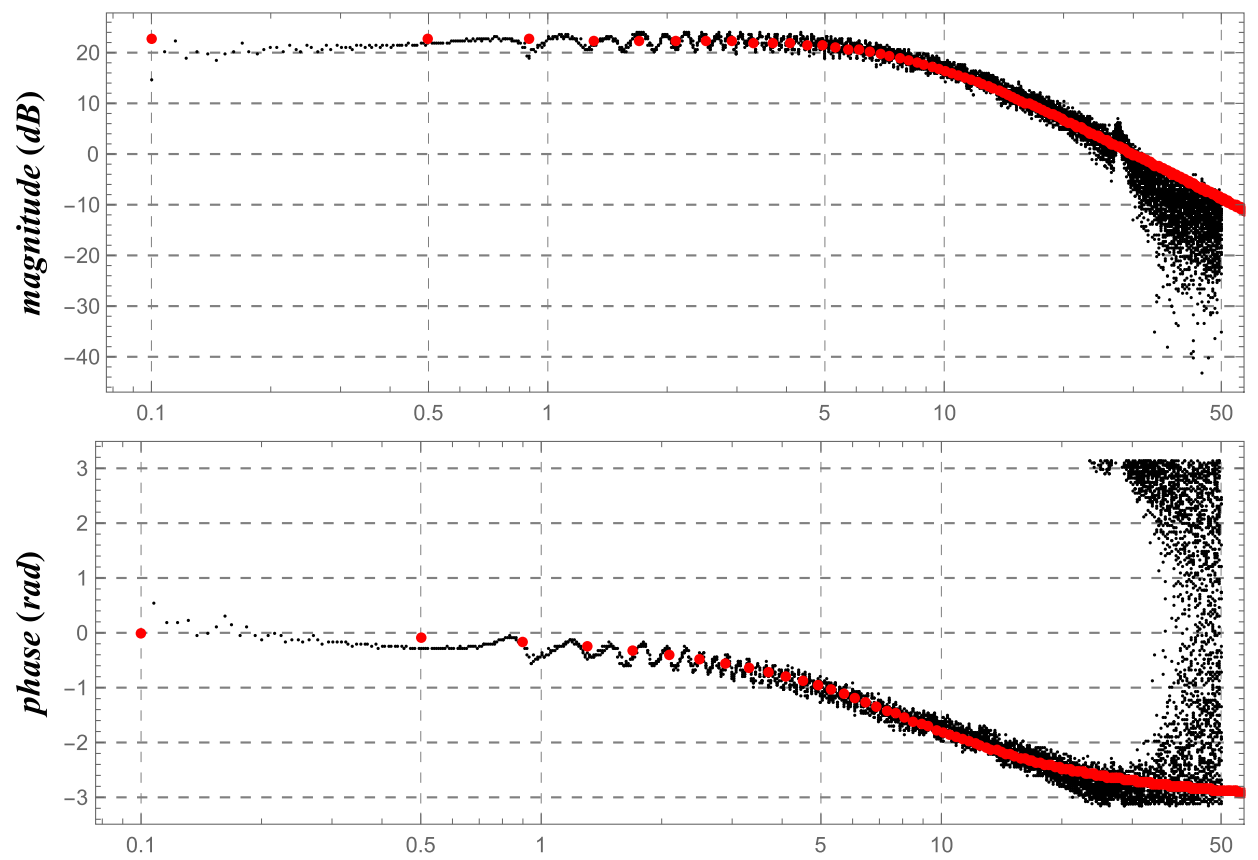

Figure 4.9 The Fitting Transfer Function by Nonlinear Fitting Method

By plotting point cloud, we determined that the system parameters (4 dimensional vector sets) could pass through a two dimensional surface $\left(2^{\text {nd }}\right.$ order function in $\left\{\lambda_{1}, \lambda_{2}\right\}$ in our program) whose mathematical expression (Eq. (3.3)) is obtained by uncertainty model with the GA method described earlier. 


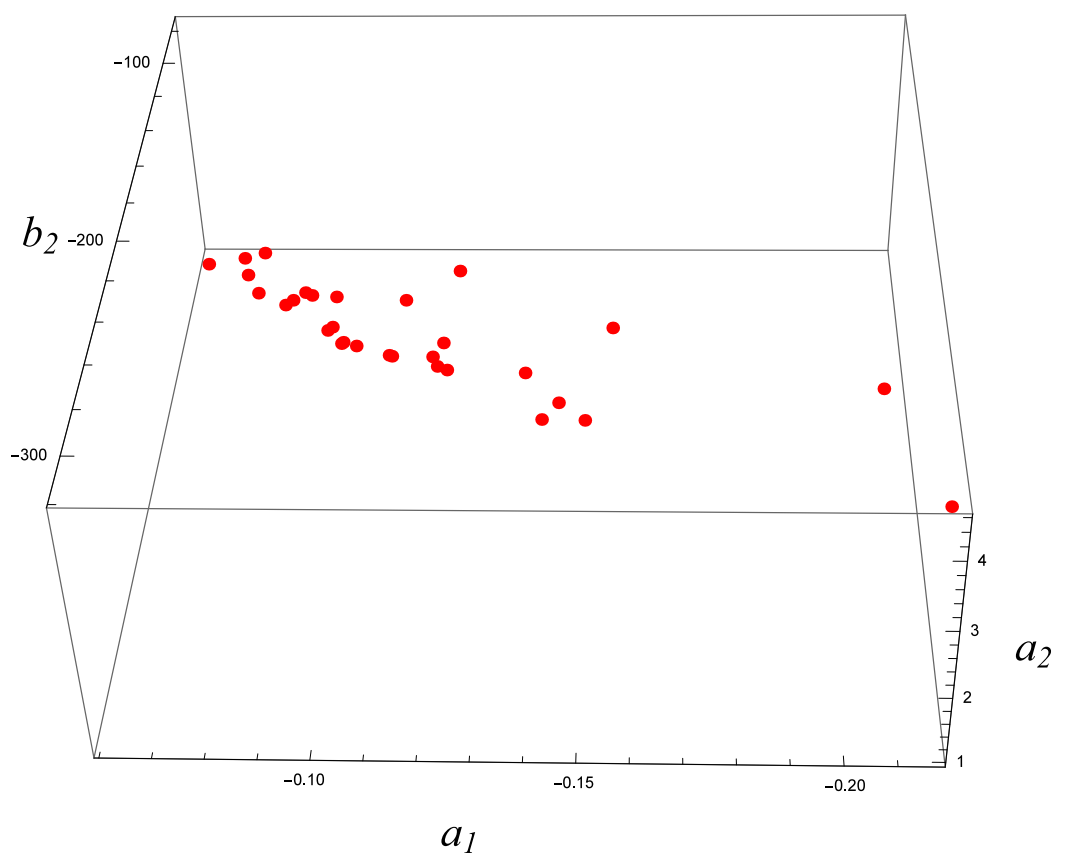

Figure 4.10 The Plot of Point Cloud for the Data Set

The result shown as follows.

$$
f(\lambda)=\left[\begin{array}{ccc}
-806.706 & -198.789 & 794.859 \\
-0.741 & 0.126 & 0.573 \\
-11.436 & -3.195 & 11.706 \\
-58.509 & -15.279 & 58.842
\end{array}\right]\left\{\begin{array}{l}
1 \\
\lambda_{1} \\
\lambda_{2}
\end{array}\right\}
$$

And the value of its corresponding $\lambda$ 's is shown in the plot. 


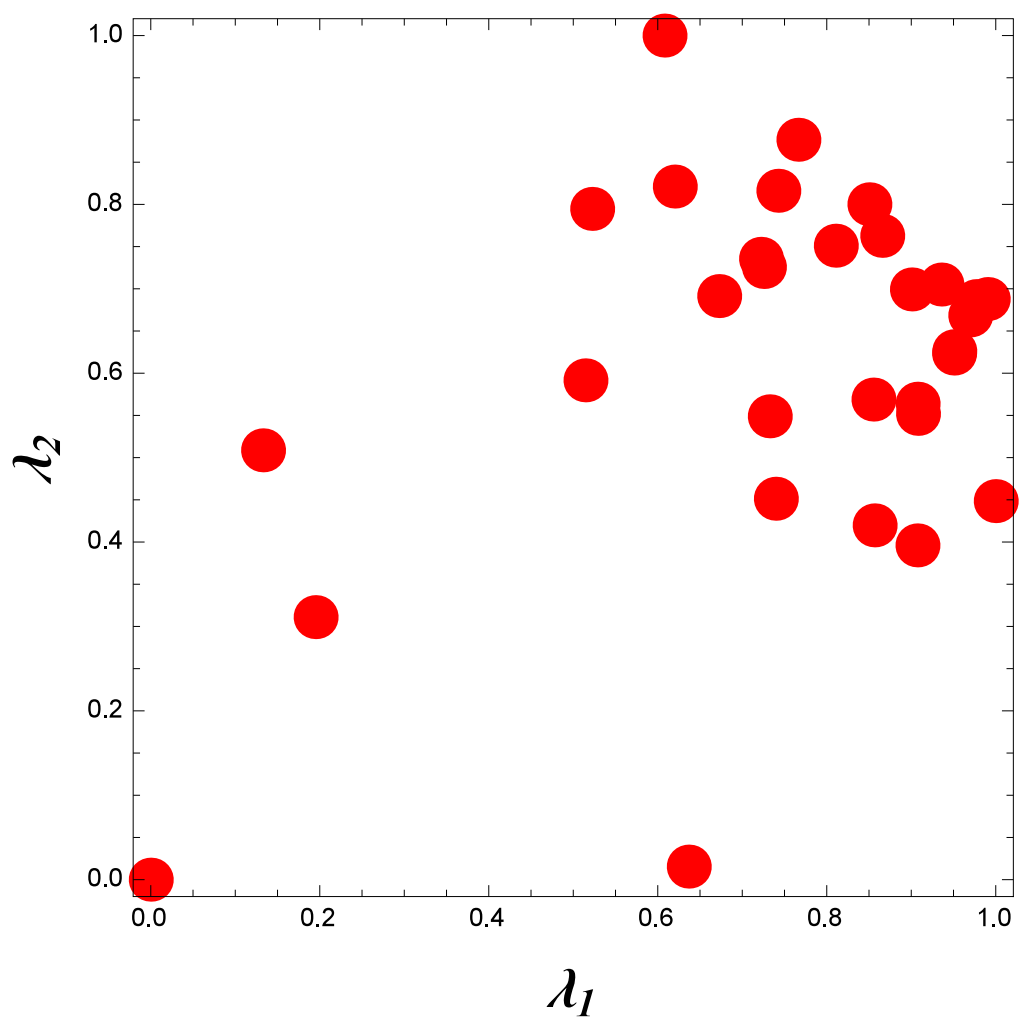

Figure 4.11 Value of $\lambda$ on a Plane

Since the uncertainty variable values has some distributions (sampling by evenly giving numbers within the range of $\lambda$ may not fully reflect the group of transfer functions), one way to estimate the distribution of the uncertainty variables is to create the kernel mixture distribution. The kernel mixture distribution is one type of smoothed density function, which assumes each data has the Gaussian function (normal distribution function) distribution impact around its neighborhood that enable expressing the impact of the density for each data. Then accumulating all the effect by each of the data by making a summation of all Gaussian functions to create a density function of distribution, which called kernel mixture distribution. 
Then the distribution function is shown in plot:

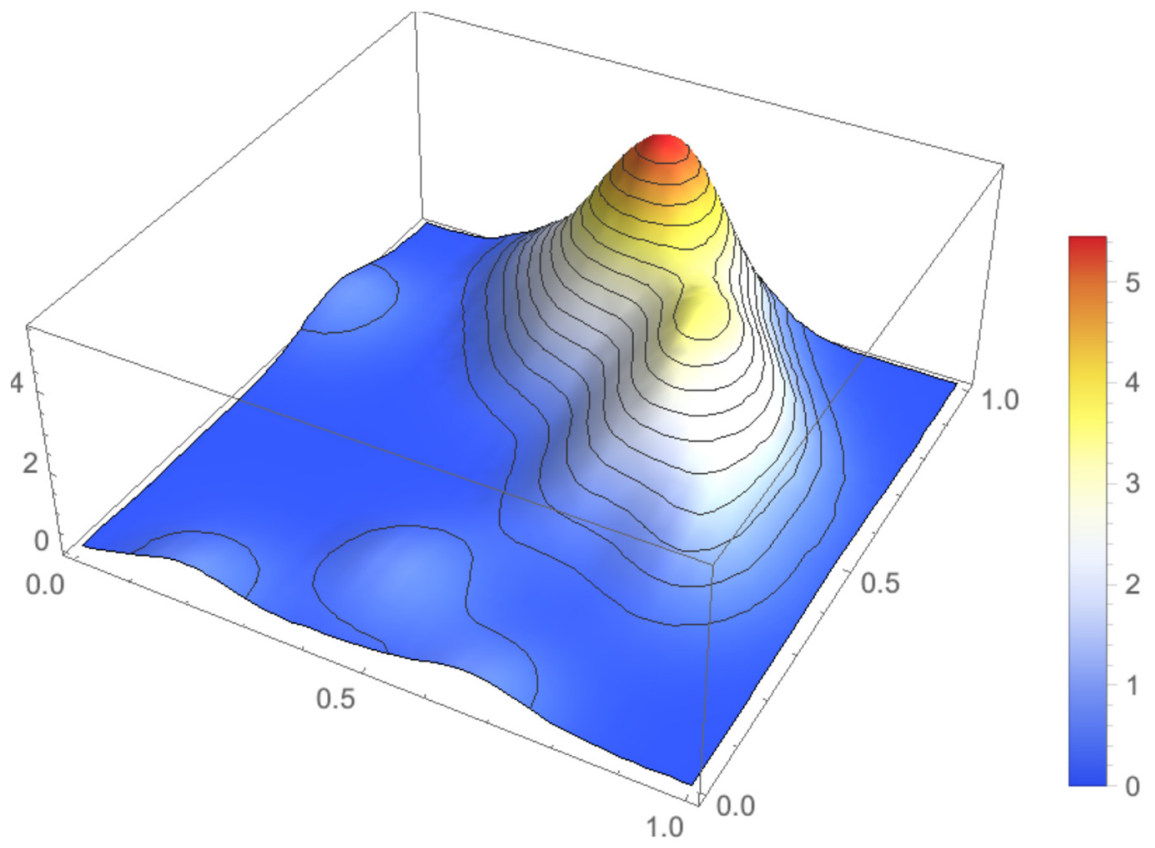

Figure 4.12 The distribution function of the uncertainty variables

Then, resampling the data by randomly generating with this distribution shown in Figure 4.12. 


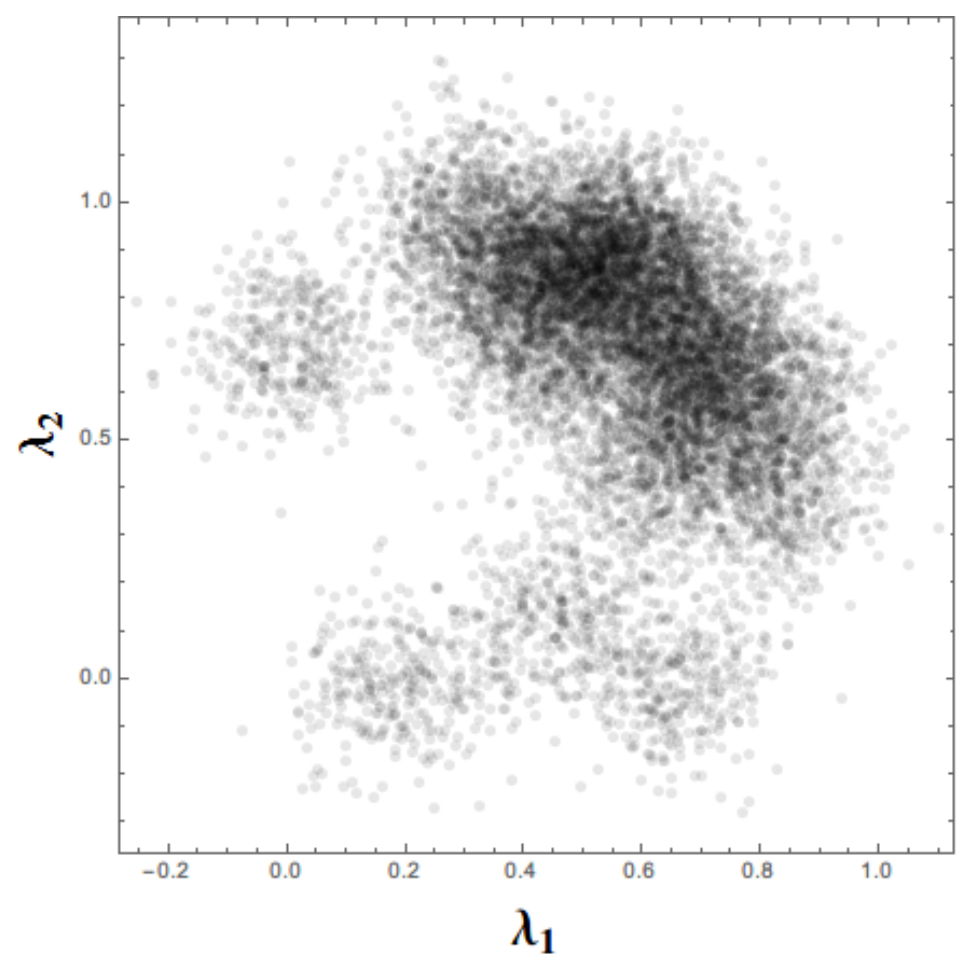

Figure 4.13 The Re-Sampled Data Set

To validate this method is to compare the frequency response of these resampled data to the frequency response of the testing data. The Bode plot of the transfer function family is,

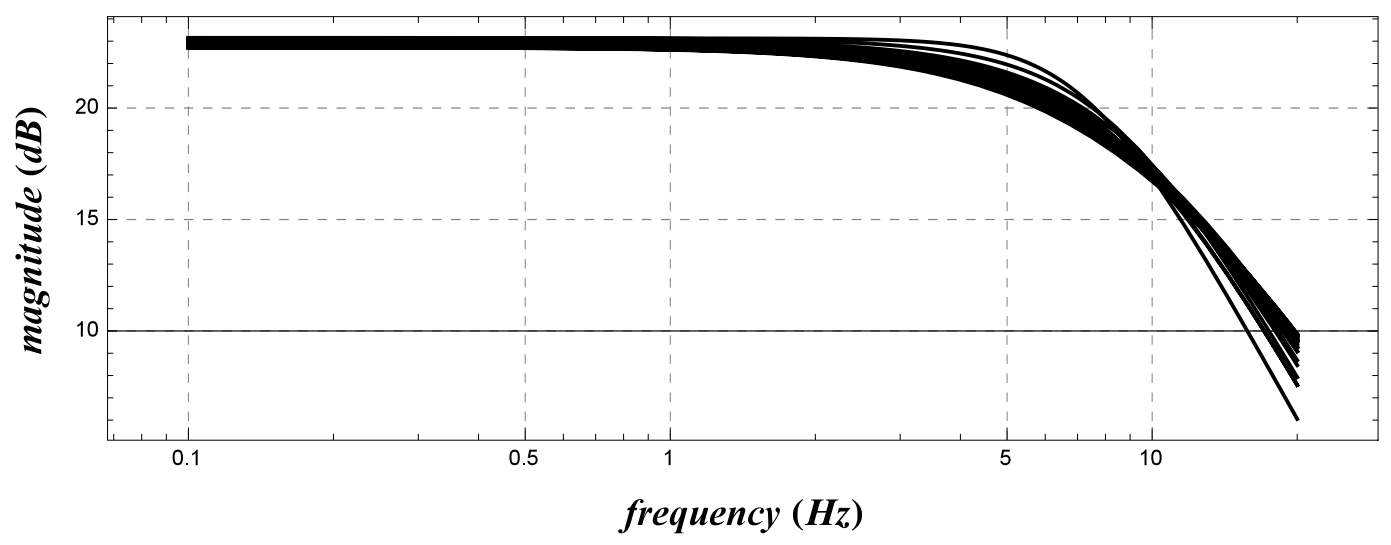




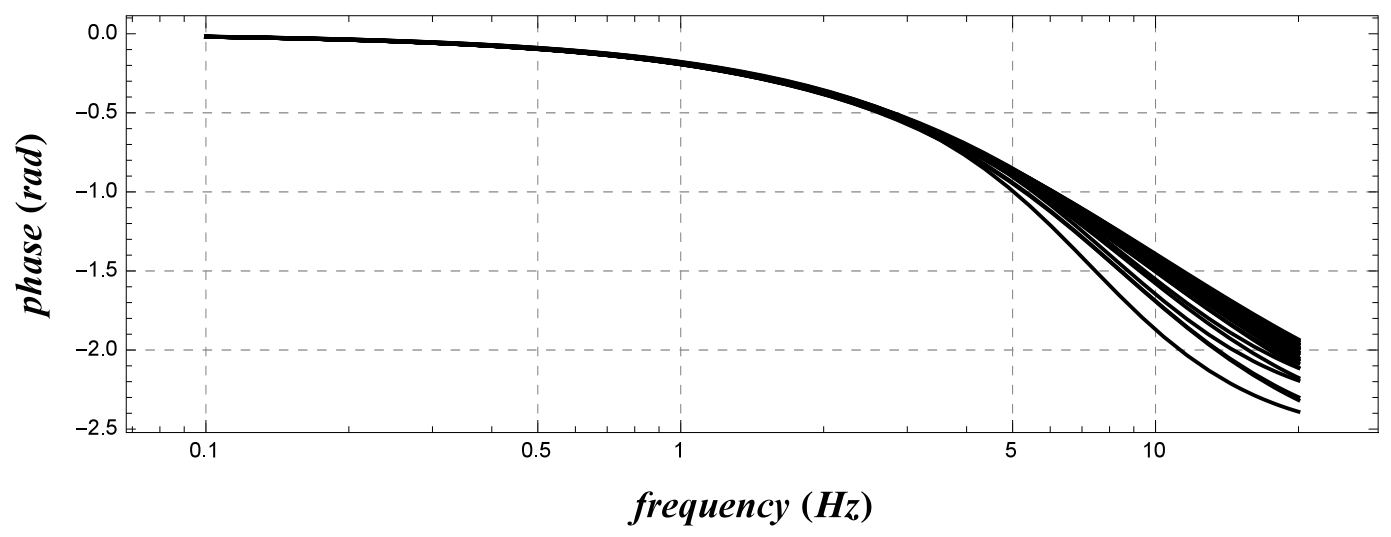

Figure 4.14 The Frequency Response of All the Fitting Data Set

The frequency response of the transfer function for the resampled uncertainty variable data set.
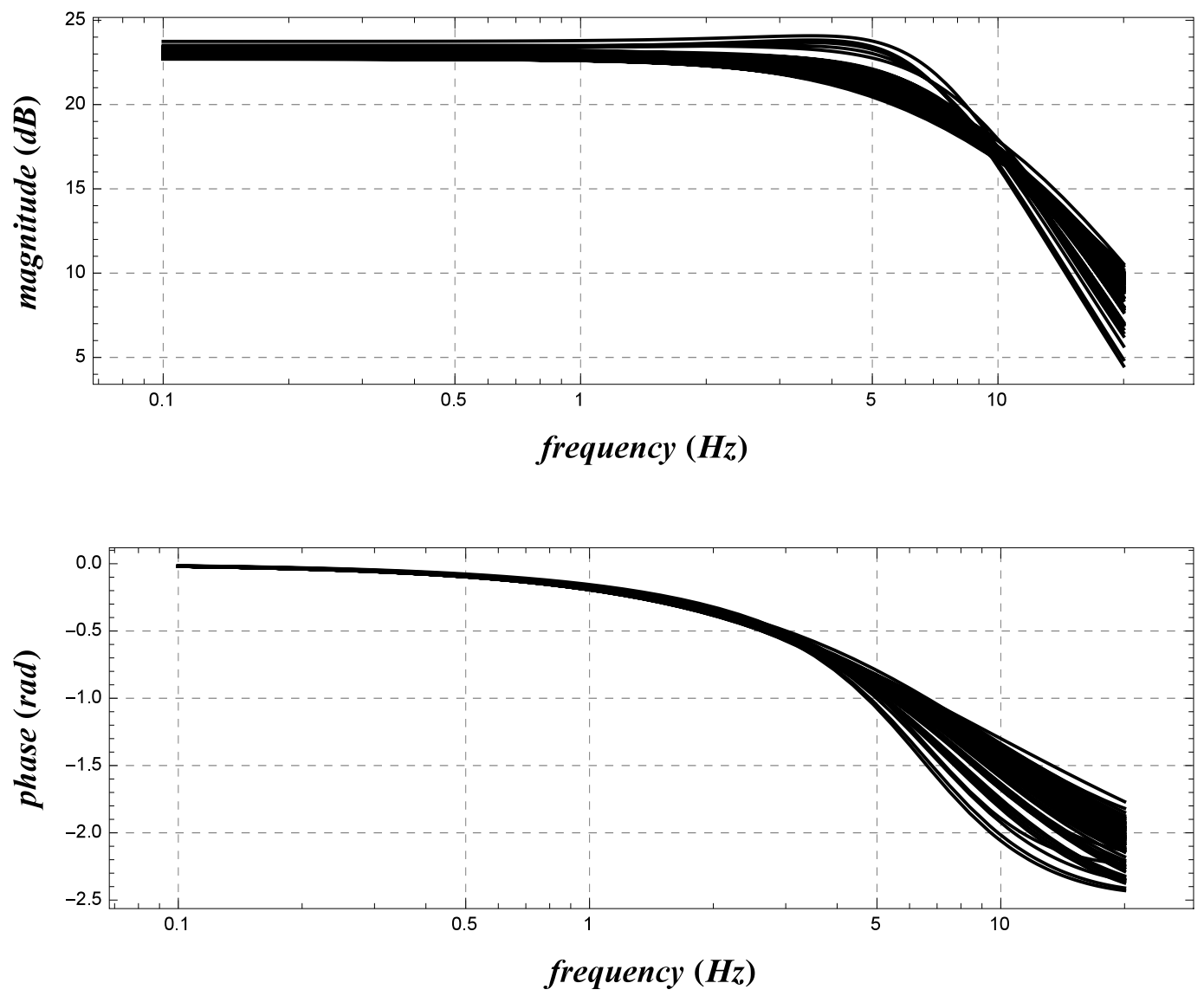

Figure 4.15 The Bode Plot of All the Re-Sampled Data Set 
These two plots (Figure 4.14 and Figure 4.15) look similar. It means the resampled data set can reflect and predict the original data at more range, which validate our assumption. However, there are no effective way to quantify the similarity between two transfer functions.

In order to compare two methods, we will apply the same close-loop system (the block diagram shown in Figure 4.6), and compare the closed-loop poles plot the of this case (shown in Figure 4.16) and previous plot (shown in Figure 4.7) with the same constant gain $K=k_{0}=2.02$.

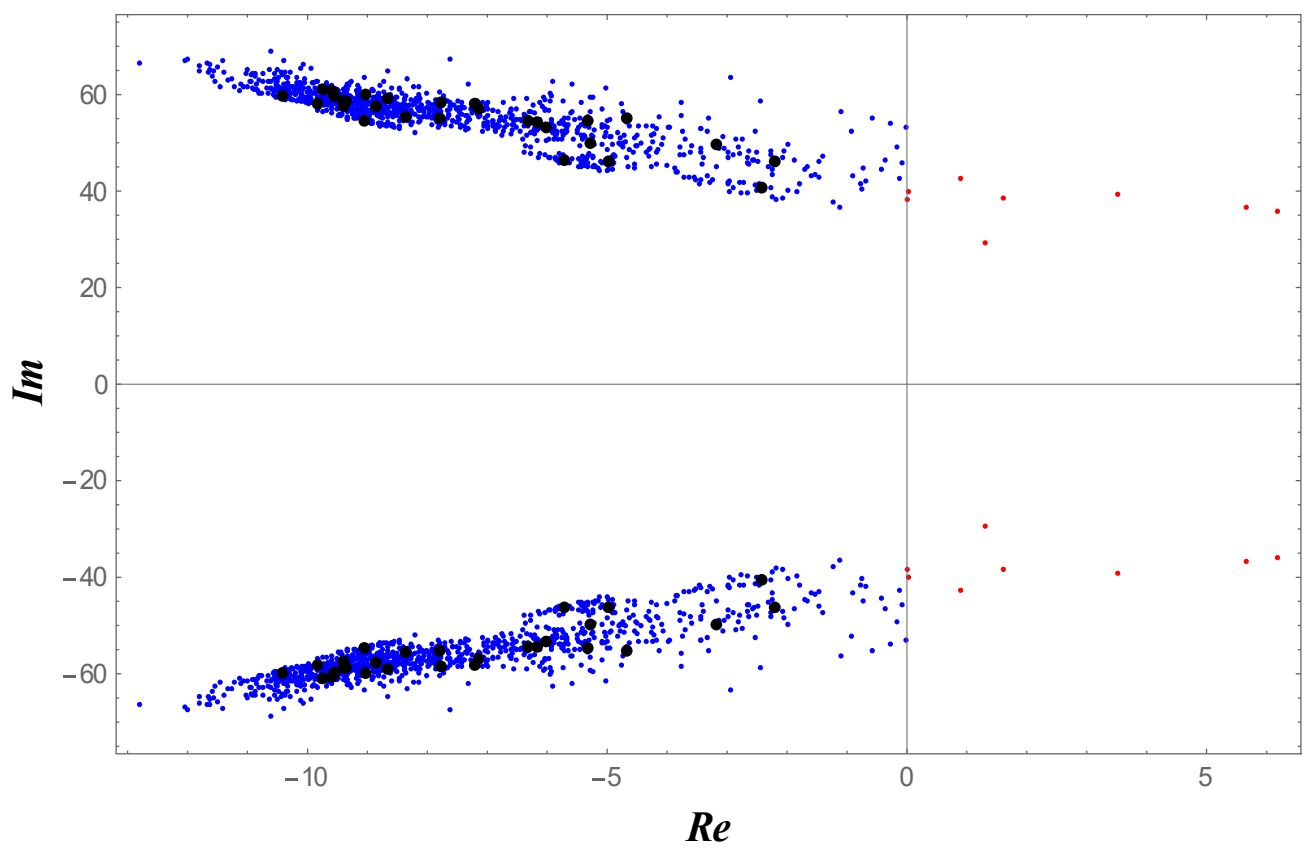

Figure 4.16 The Closed-loop Poles of the Resampled Transfer

Functions at $K=k_{0}=2.02$

The plot shows that the closed-loop poles of experimental thirty plants (black dots) and the majority of resampled transfer functions (blue dots) are in the left half plane - stable or marginal stable. Although most cases are stable, 
however there are $1.24 \%$ (red dots; unstable case) at the right half plane. Since it's a statistics process and a marginal stable case from previous analysis, few solutions that are not stable is acceptable. Nevertheless, this result validates the correctness of our previous analysis.

\subsection{H-Infinity Stability}

Another way of checking the stability of the control system is to check the H-Infinity norm, i.e. to check whether the infinity norm of the control system closed-loop transfer function (described later) in any frequency is less than or equal to 1.

Firstly, picking one plant as nominal plant $G_{0}(s)$, then calculating the multiplicative error $E(s)$ of the transfer function family generate by resampled model.

The multiplicative error $E(s)$ is defined as:

$$
E(s)=\left|\frac{G(j \omega)-G_{0}(j \omega)}{G_{0}(j \omega)}\right|
$$

Where the transfer function of selected nominal plant is:

$$
G_{0}(s)=\frac{-493.205+s}{-35.756-7.043 s-0.29 s^{2}}
$$




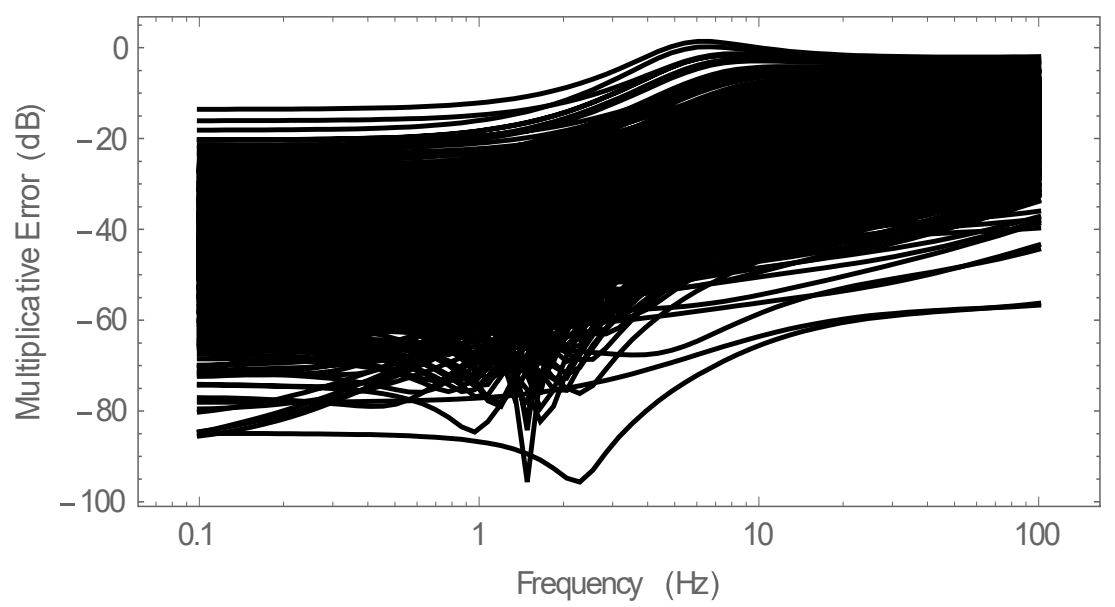

Figure 4.17 The Family of Transfer Functions for Multiplicative Error

From the family of transfer functions for multiplicative error (shown in Figure 4.17), we captured the maximum of multiplicative error (shown in Figure 4.18) to check the stability of maximum multiplicative error case:

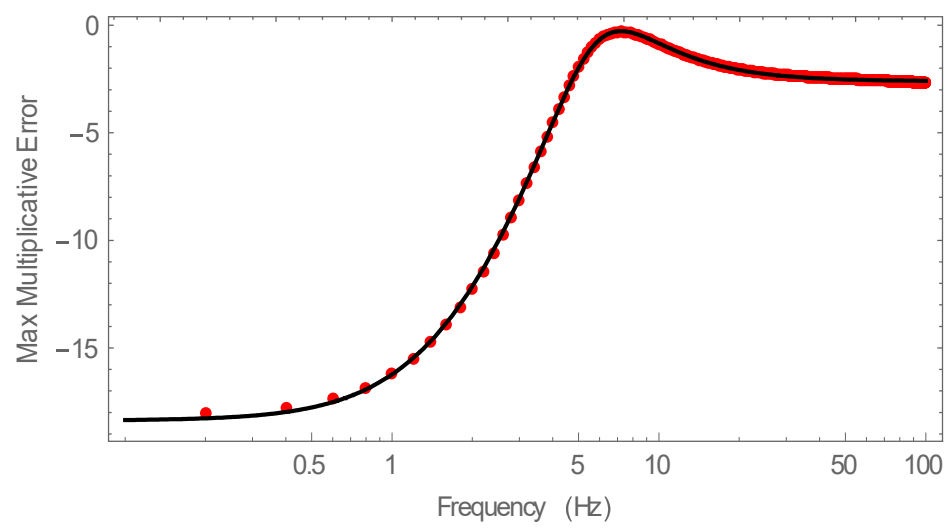

Figure 4.18 The Transfer Function Fit for Multiplicative Error $W(s)$

The Transfer Function Fit for Maximum Multiplicative Error is $W(s)$ :

$$
W(s)=\frac{-5.51-2.43 s+s^{2}}{45.67+7.91 s+1.35 s^{2}}
$$


To ensure the stability that bounded all uncertainties of multiplicative error, we need to check the infinity norm of the transfer function for the feedback control system: $\left\|\frac{G_{0}(j \omega) K}{1+G_{0}(j \omega) K} W\right\|_{\infty} \leq 1, \forall \omega$. The block diagram of feedback controller with multiplicative error form is shown in Figure 4.19.

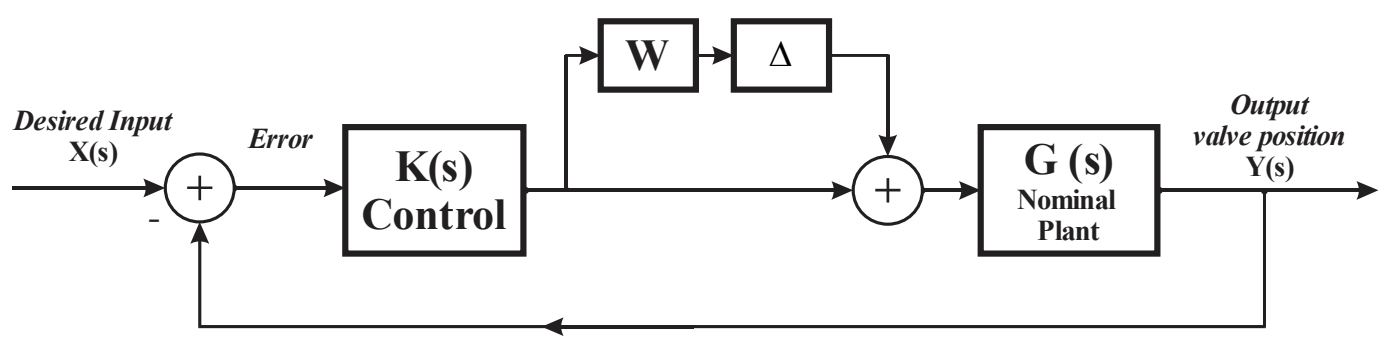

Figure 4.19 Feedback Control with Multiplicative Error Form

Then we apply previous result $K=2.02$ and we can get:

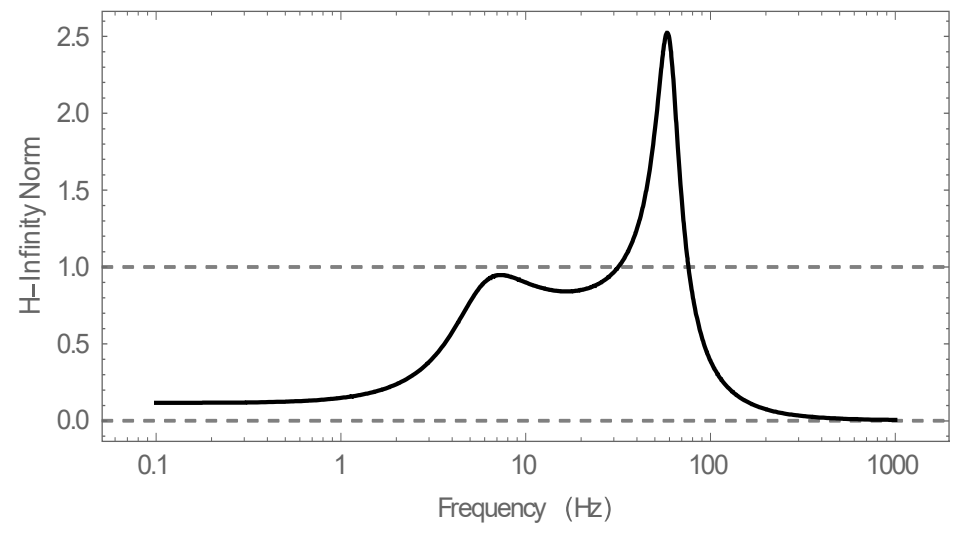

Figure 4.20 The Norm Function of the Transfer Function at $K=2.02$

The infinity norm of $\frac{G_{0}(j \omega) K}{1+G_{0}(j \omega) K} W$ is 2.53 , which is unstable at $20 \sim 80$ Hz. We can then optimize constant gain $K$ at $K=0.53$ to satisfied the $\mathrm{H}$ infinity norm equals 1 for all frequencies, shown in Figure 4.21. 


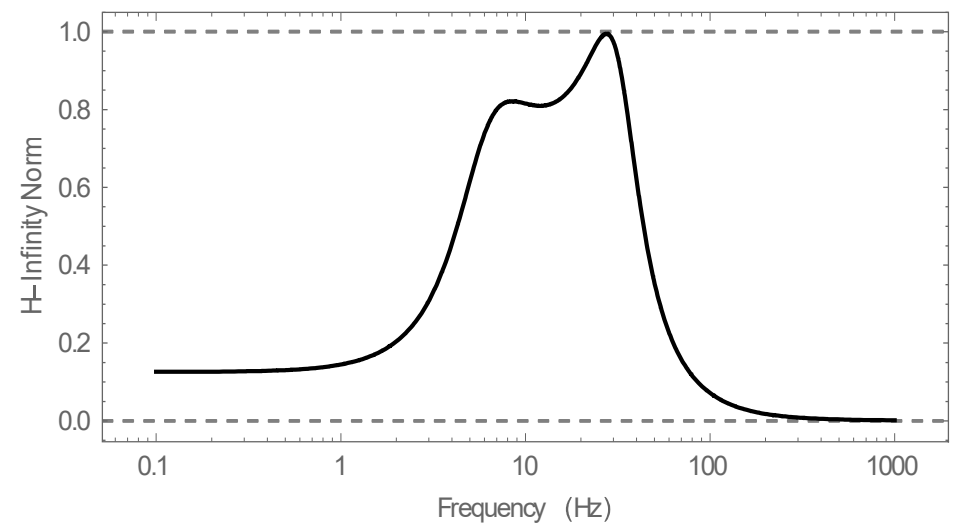

Figure 4.21 The Norm Function of the Transfer Function at $K=0.53$

Then the closed-loop poles can perfectly include at left half plane, which means the system is stable.

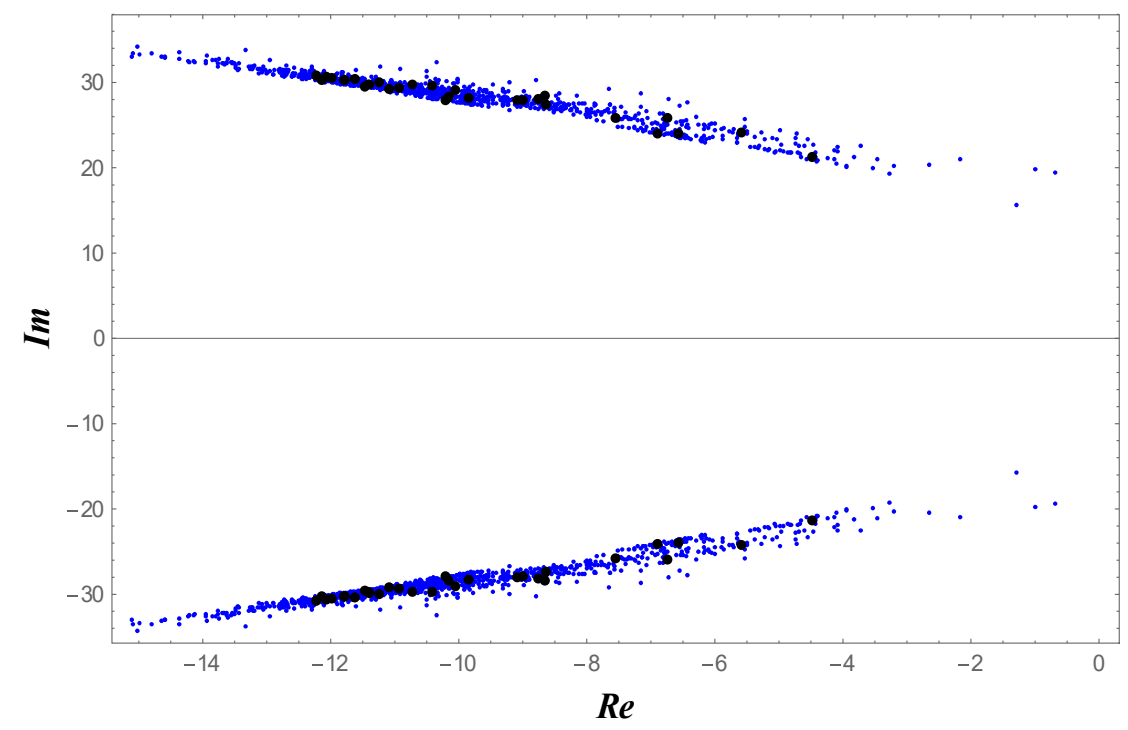

Figure 4.22 The Closed-loop Poles of the Resampled Transfer

Functions at $K=k_{0}=0.53$

\subsection{Limitation of Models}

During the research of uncertainty modeling, diverse optimization algorithm or chosen structures of transfer functions result in different types of data set: it 
firstly appears to be one dimensional line, then a plane. When choosing different structures or higher order transfer functions, the data type varies in unpredicted ways. However, this uncertainty works in successfully searching the relations in existing data set where the optimization algorithm cannot completely regress and do well with the reduction of uncertainties without knowing the uncertainty types. 


\section{CHAPTER 5: DIMENSION SEARCH CONCEPTUAL DESIGN}

\subsection{Overview of Dimension Search Method}

The type of the data set of this uncertainty model is assumed to be low dimensional continuous geometries that can cover most of the data. However, since the dimension properties of the point cloud and its corresponding geometry is ambiguous, the dimension searching method is needed.

The dimension property of entire the data set can be measured by the dimension properties of small neighborhoods. Then following this idea by assuming that the dimension properties of the whole geometry are depending on measuring the dimension properties of the smallest neighborhood test geometries (called dimension test geometry) showing its properties of each place of the point cloud. In other words, the property in one-dimension can be measured by the length of the line, the two-dimension property can be measured by the area of triangle, and the three-dimension property can be measured by the volume of the tetrahedron, etc. Let's take an example. If the data set approximately on the onedimension, it can be shown as a high dimension lines by one-dimension test geometry - lines. 


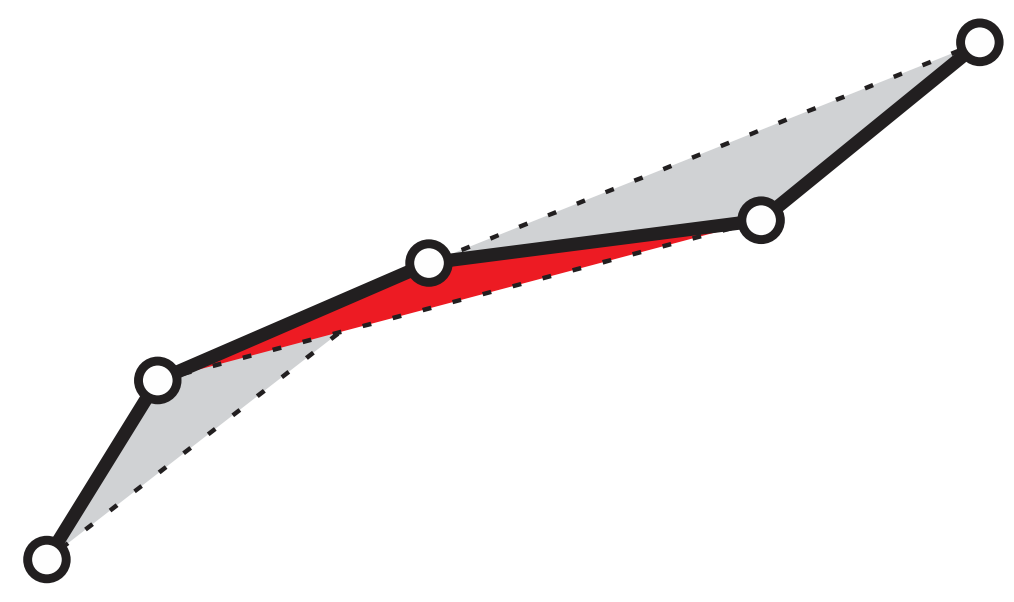

Figure 5.1 Example of 1-D Properties of Data Set

We can see that the area of each triangle is relatively small. Nevertheless, the length of the point is large in this example shown in Figure 5.1. Thus, this data set is recommended to be expressed in one dimension.

\subsection{Conceptual Design of Dimension Search Algorithm}

In this conceptual design, we divide the measurement of the dimension properties into measuring dimension properties of the dimension test geometries around the neighborhood. The measuring of the dimension properties is defined by "Cayley-Menger Determinant" [6], which can determine property of the dimension test geometries.

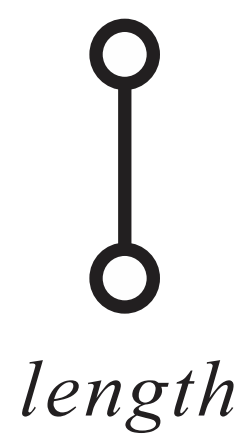

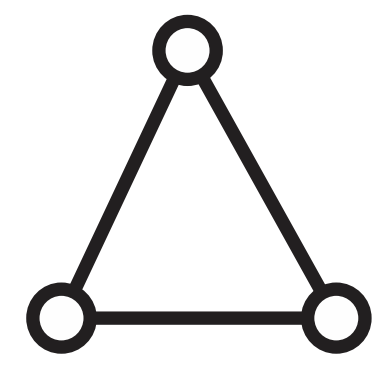

area

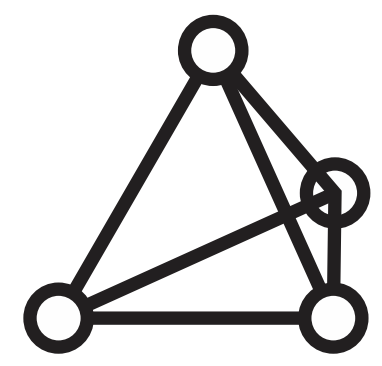

volume 


\section{Figure 5.2 The Dimension Test Geometry}

The $1 \mathrm{D}$ property will determine by at least 2 points, $2 \mathrm{D}$ is 3 points, $3 \mathrm{D}$ is 4 points, etc.

Firstly, numbering the data set by giving each data an index number. Secondly, calculate the distance between each two points, such as the distance between in $i^{\text {th }}$ and $j^{\text {th }}$ point is written as $\delta_{i j}$.

$$
\delta_{i j}=\left\|p_{i}-p_{j}\right\|
$$

Where $p_{i}$ is the data (can be regarded as coordinate point), the norm is 2

norm.

Then the Cayley-Menger Determinant is written by this form:

$$
\Delta_{k}=\sqrt{\frac{(-1)^{k-1}}{2^{k}(k !)^{2}} \cdot\left|\begin{array}{l:c}
\mathbf{0} & \mathbf{1} \\
\hdashline \mathbf{1} & {\left[\delta_{i j}^{2}\right.}
\end{array}\right|}
$$

Such as the volume of the general tetrahedron is:

$$
\Delta_{3}=\sqrt{\frac{1}{288} \cdot\left|\begin{array}{c:cccc}
0 & 1 & 1 & 1 & 1 \\
\hdashline 1 & 0 & \delta_{12}^{2} & \delta_{13}^{2} & \delta_{14}^{2} \\
1 & \delta_{21}^{2} & 0 & \delta_{23}^{2} & \delta_{24}^{2} \\
1 & \delta_{31}^{2} & \delta_{32}^{2} & 0 & \delta_{34}^{2} \\
1 & \delta_{41}^{2} & \delta_{42}^{2} & \delta_{43}^{2} & 0
\end{array}\right|}
$$

Where $\Delta_{k}$ is the measurement of the dimension test geometry, which shows the dimension properties of the geometry; in other words, when $k=1$, it denotes 
the length of line $\Delta_{1}$, when $k=2$, it denotes the area of triangle $\Delta_{2}$, when $k=3$, it denotes the volume of tetrahedron $\Delta_{3}$, etc.

The way of measure the dimension property of the global case is still controversial and not well established. One way of building it is to consider the dimension property for each data and its neighborhood and to measure the accumulation of the dimension properties globally. Namely, we can establish a small test geometry of each data and its neighborhood and calculate the measurement for small test geometries, and then getting the global measurement by accumulating all the measurement for all data set.

The global measurement algorithm can be this form (conceptual design).

$$
M_{k}=\left(\sum_{i} \Delta_{k}^{(i)}\right)^{\frac{1}{k}}
$$

Where the $M_{k}$ is the measurement of $k^{\text {th }}$ dimension property, $i$ here is the index of each data. $\Delta_{k}^{(i)}$ is the $k^{\text {th }}$ dimension property of $i^{\text {th }}$ data geometry. $M_{k}$ means the ratio between the accumulation of measurement on the dimension test geometry projected into one dimension and the space that the point cloud take up projected into one dimension (since the model has been normalized, this term will be the unit length -1$)$.

\subsection{Examples and Results of Dimension Search Algorithm}

Let's build several examples to illustrate my conceptual design. 


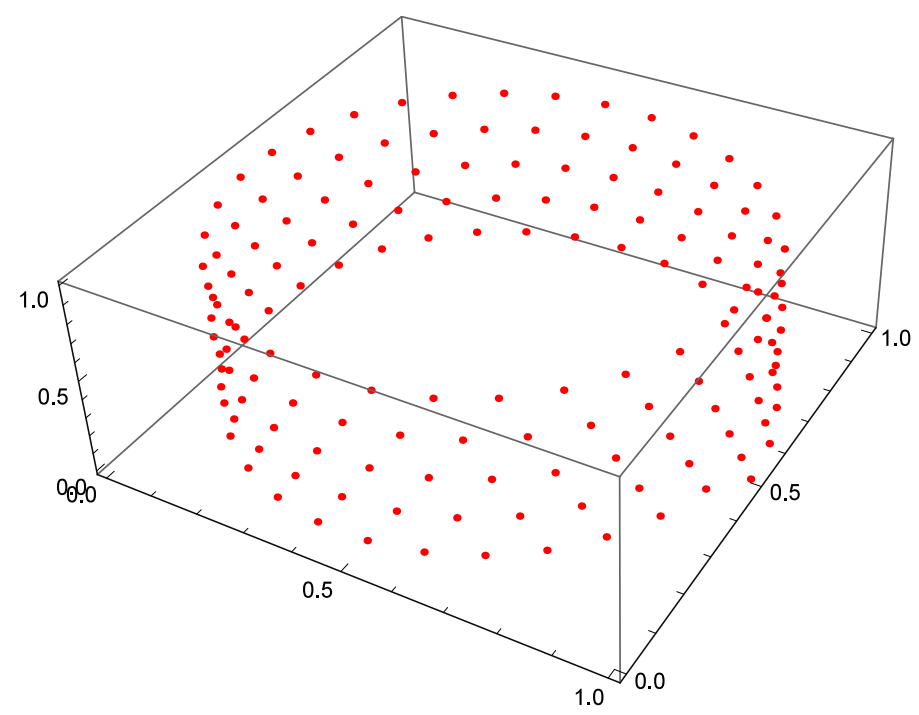

Figure 5.3 The Example of 3-D Helix Curve

The data will be initially normalized to the range of $(0,1)$ by the normalization method in Eq. (2.3). Then, establish dimension test geometries individually for each data until finish building for all data, the result is shown in the plot.

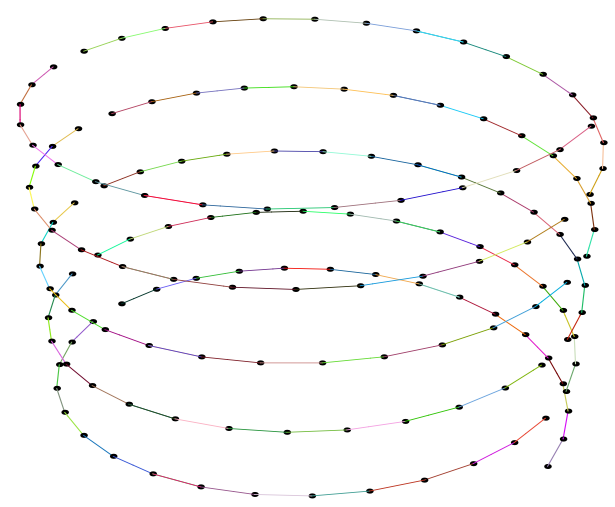

Figure 5.4 1-D Property of 3-D Helix Curve 
The global measurement for $1-\mathrm{D}$ is $M_{1}=15.80667$.

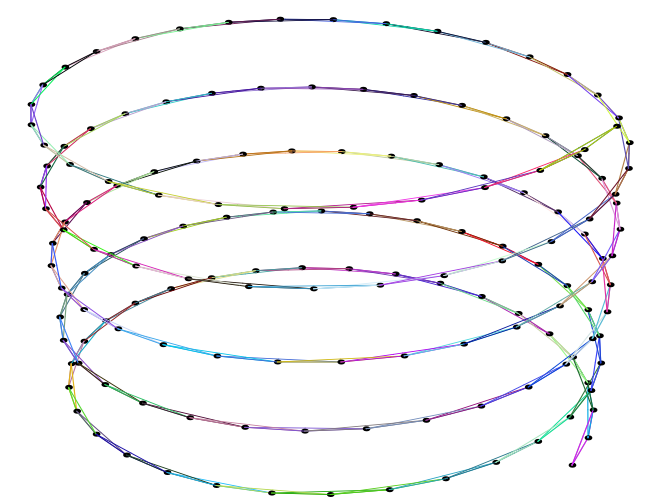

Figure 5.5 2-D Property of 3-D Helix Curve

The global measurement for 2-D is $M_{2}=0.39594$.

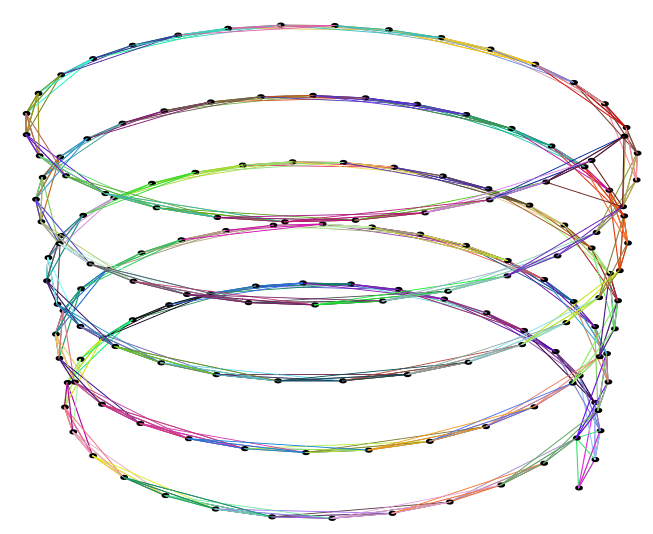

Figure 5.6 3-D Property of 3-D Helix Curve

The global measurement for 3-D is $M_{3}=0.05255$. 
The dimension property in 1-D is relatively large, yet in 2 or 3-D are small. Thus the data point is recommended to denoted to be 1-D problem.

However, if there are less samples in the same helix, the result is entirely different.

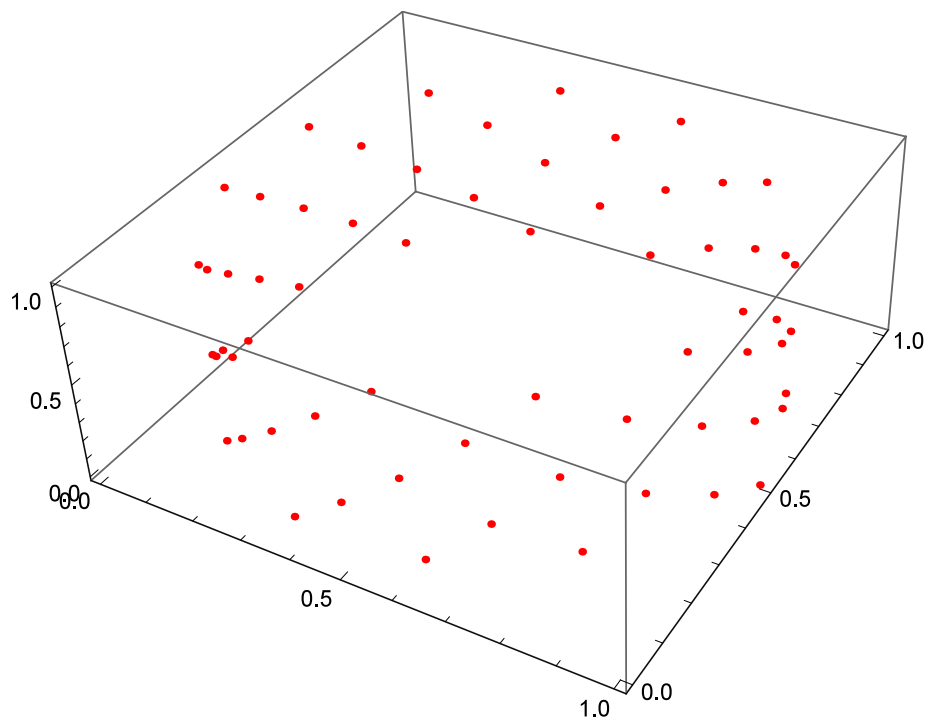

Figure 5.7 The Example of 3-D Helix Curve with Less Sample

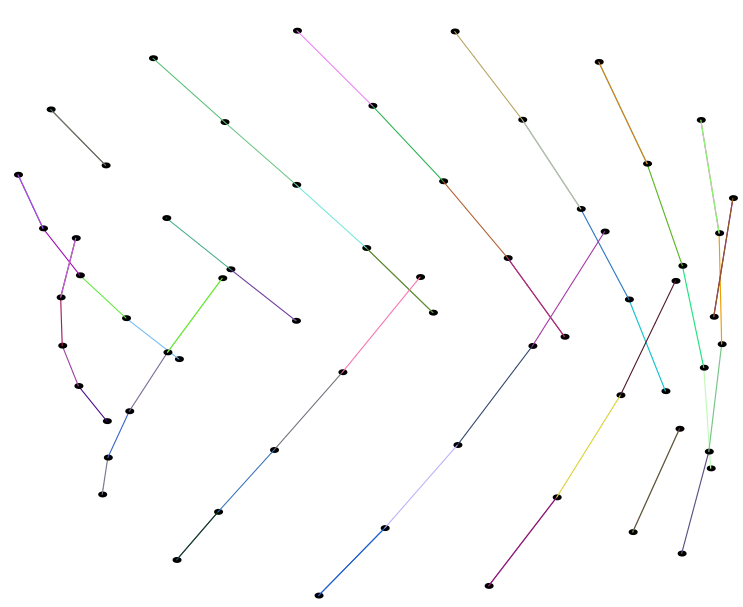




\section{Figure 5.82 1-D Property of Less Sampled 3-D Helix Curve}

The global measurement for 1-D is $M_{1}=14.86648$.

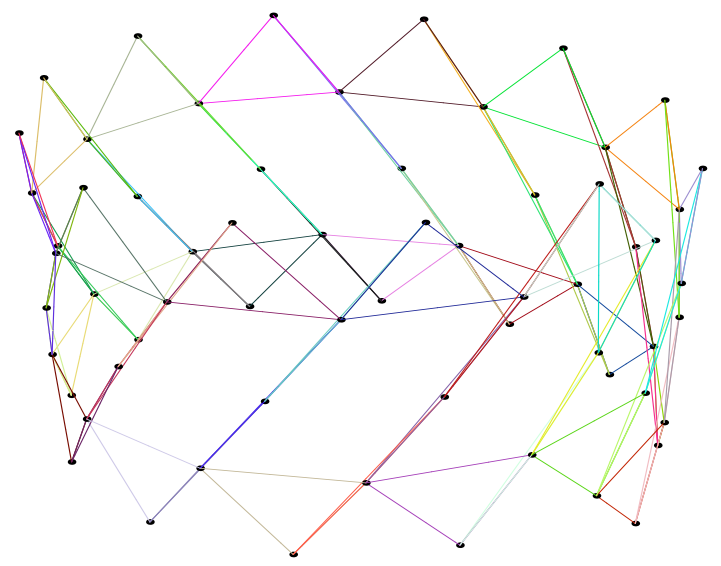

Figure 5.9 2-D Property of 3-D Helix Curve

The global measurement for 2-D is $M_{2}=0.86975$.

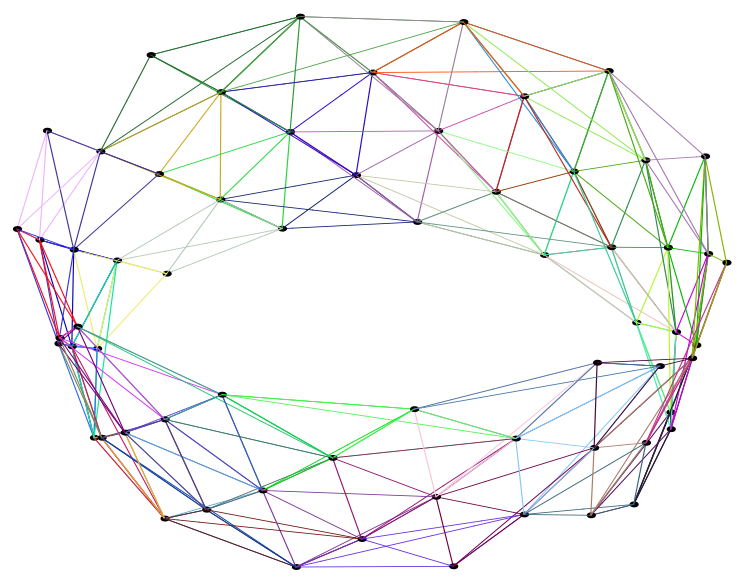




\section{Figure 5.10 3-D Property of 3-D Helix Curve}

The global measurement for 3-D is $M_{3}=0.27291$.

The dimension property in 1 or 2-D is relatively large, yet 3-D are relatively small. Thus the data point is recommended to denoted to be 2-D problem.

For the data set for the valve (shown in Figure 4.10), the result is:

$$
\left\{\begin{array}{l}
M_{1}=2.17882 \\
M_{2}=0.35768 \\
M_{3}=0.000509
\end{array}\right.
$$

Since the 3-D property is small, it can be 1-D or 2-D problem for this case.

However, the effective value or algorithm for the measurement is still unknown, the current measurement value is not good enough to directly see the dimension properties, which will be the future work for us to develop. 


\section{CHAPTER 6: CONCLUSIONS}

A new method of determining a parameterization of an uncertainty model has been presented. Since the polynomial optimization problem is sometimes nonconvex, the use of the genetic algorithm to overcome the problem of non-convex optimization is adopted in our research work. Then, we proposed normalization method and series of coefficient to improve the effectiveness of the algorithm and the fidelity of the data in genetic algorithm. The conceptual design of the dimension search algorithm straightforwardly shows the dimension properties of a data set in high dimension space.

The methods developed in this work were applied to an electrohydraulic valve in a close loop control system. The uncertainty of this LTI model of the valve system containing four system parameters was firstly parameterized using only one variable, then using another nonlinear complex optimization method with two variables for comparison. With this simple parameterization, we have shown that a parameterized model can be used to determine the range of a controller parameter to obtain robust stability of a closed loop control system using a root locus technique. The refined model by adopting a better complex value optimization method by using the norm function with one starting point, then using the resampling technique by introducing the mix-kernel distribution is

presented to be better estimate the density of data and achieve better result. Two techniques in comparison validate the correctness of our model.

This thesis has present four contributions: 
1. Designed normalization method of linear transformation to achieve better performance of GA;

2. Determined transfer function of dynamic response by applying FFT and nonlinear complex fitting method;

3. Created kernel-mix distribution of resampling method to generate series of transfer function family;

4. Built a dimension search concept design method to show reduced dimension properties of the point cloud using Cayley-Menger Determinant. 


\section{CHAPTER 7: $\quad$ FUTURE WORK}

Our work suggests several interesting future directions:

(1) Because this uncertainty model is based on the lower dimension geometries homomorphism to an open ball, if the data sets can be in other topologies, a specific transformation in Cartesian space can be applied on the polynomial to form a featured topology - and this problem can also be solved by GA.

(2) In our example, we only give the constant gain $K$ in the close loop. If $K(s)$ can be a transfer function, or even using different control type, this parameterized uncertainty model can also be applied into it.

(3) There are no uniform criteria for the dimension properties in the dimension search algorithm, which can show the dimension properties more accurately.

(4) A delicate design of the dimension search algorithm is needed to show the dimension properties of the point cloud. 


\section{REFERENCES}

[1] S. Skogestad, Postlethwaite, I, "Multivariable Feedback Control," ed. West Sussex, England: John Wiley and Sons Ltd., 2005.

[2] R. Carpenter and R. Fales, "Mixed Sensitivity H-Infinity Control Design with Frequency Domain Uncertainty Modeling For a Pilot Operated Proportional Control Valve," in ASME 2012 5th Annual Dynamic Systems and Control Conference joint with the JSME 2012 11th Motion and Vibration Conference, 2012, pp. 733-741.

[3] M. Sepasi, F. Sassani, and R. Nagamune, "Parameter uncertainty modeling using the multidimensional principal curves," Journal of Dynamic Systems, Measurement, and Control, vol. 132, p. 054501, 2010.

[4] R. Conway, S. Felix, and R. Horowitz, "Model reduction and parametric uncertainty identification for robust H 2 control synthesis for dualstage hard disk drives," Magnetics, IEEE Transactions on, vol. 43, pp. 3763-3768, 2007.

[5] E. A. Yazdi, M. Sepasi, F. Sassani, and R. Nagamune, "Automated multiple robust track-following control system design in hard disk drives," Control Systems Technology, IEEE Transactions on, vol. 19, pp. 920-928, 2011.

[6] M. J. Sippl and H. A. Scheraga, "Cayley-menger coordinates," Proceedings of the National Academy of Sciences, vol. 83, pp. 2283-2287, 1986. 
[7] J. Clegg, J. F. Dawson, S. J. Porter, and M. H. Barley, "The use of a genetic algorithm to optimize the functional form of a multi-dimensional polynomial fit to experimental data," in Evolutionary Computation, 2005. The 2005 IEEE Congress on, 2005, pp. 928-934.

[8] M. Gulsen, A. Smith, and D. Tate, "A genetic algorithm approach to curve fitting," International Journal of Production Research, vol. 33, pp. 19111923, 1995.

[9] R. Carpenter, R. Fales, "Proportional control system design and probability of stability for a pilot operated proportional valve with parametric uncertainty," presented at the Symposium on Fluid Power and Motion Control, Bath, UK, 2012. 


\section{VITA}

Zuheng Kang lived in Xiangyang, Hubei, China where he finishes almost all education level until Bachelor Degree. Then came to University of Missouri Columbia to finish master of science degree. He graduated from the Hubei University of Arts and Science with BS in Mechanical Engineering with an emphasis in Automobile and Manufacturing. He receives MS in mechanical and aerospace engineering at the University of Missouri - Columbia in May 2016. Throughout his undergraduate and graduate careers, he has focused on studies in Automobile, Manufacturing, dynamics, controls, and optimization. In his study, he publishes part of this thesis as ASME conference paper and presented in Columbus, Ohio with Dr. Fales.

In addition to study engineering, Zuheng Kang (also named Robert Bogan Kang) is an avid music performer, who can play well in violin, piano, baroque recorder, flute, harmonica, whistle and create realistic synthesized performance with various software. He also dedicated into music performance and composition theory research works including Frequency Component Analysis for music composition, rhythm structure reconstruction, psycho-acoustics, performance technique, auditory and optic nerves modeling, overtones properties of musical instruments using Finite Element Methods. Besides he enjoys providing piano tuning and fixing volunteering works for friends and church and playing badminton with teammates. 